\title{
Fractional Sobolev Spaces via Riemann-Liouville Derivatives
}

\section{Dariusz Idczak and Stanisław Walczak}

Faculty of Mathematics and Computer Science, University of Lodz, Banacha 22, 90-238 Lodz, Poland

Correspondence should be addressed to Dariusz Idczak; idczak@math.uni.lodz.pl

Received 31 July 2013; Accepted 19 October 2013

Academic Editor: Ismat Beg

Copyright (C) 2013 D. Idczak and S. Walczak. This is an open access article distributed under the Creative Commons Attribution License, which permits unrestricted use, distribution, and reproduction in any medium, provided the original work is properly cited.

Using Riemann-Liouville derivatives, we introduce fractional Sobolev spaces, characterize them, define weak fractional derivatives, and show that they coincide with the Riemann-Liouville ones. Next, we prove equivalence of some norms in the introduced spaces and derive their completeness, reflexivity, separability, and compactness of some imbeddings. An application to boundary value problems is given as well.

\section{Introduction}

Let $1 \leq p<\infty$. Classical Sobolev space $W^{1, p}=$ $W^{1, p}\left((a, b), \mathbb{R}^{m}\right)$ of order one on an open bounded interval $(a, b) \subset \mathbb{R}$ is defined by (cf. [1])

$$
\begin{gathered}
W^{1, p}=\left\{u \in L^{p} ; \underset{g \in L^{p}}{\exists} \underset{\varphi \in C_{c}^{\infty}}{\forall} \int_{a}^{b} u(t) \varphi^{(1)}(t) d t\right. \\
\left.=-\int_{a}^{b} g(t) \varphi(t) d t\right\},
\end{gathered}
$$

where $L^{p}=L^{p}\left((a, b), \mathbb{R}^{m}\right)$ is the space of functions $g$ : $(a, b) \rightarrow \mathbb{R}^{m}$ that are integrable with power $p, C_{c}^{\infty}=$ $C_{c}^{\infty}\left((a, b), \mathbb{R}^{m}\right)$ is the set of smooth functions $\varphi:(a, b) \rightarrow$ $\mathbb{R}^{m}$ with compact support $\operatorname{supp} \varphi \subset(a, b)$, and $\varphi^{(1)}$ is the classical derivative of $\varphi$. The function $g$ satisfying the above condition is denoted by $u^{\prime}$ and called the weak derivative of $u$ of order one.

Sobolev space $W^{n, p}=W^{n, p}\left((a, b), \mathbb{R}^{m}\right)$ of order $n>1$ is defined by (cf. [1])

$$
W^{n, p}=\left\{u \in W^{n-1, p} ; u^{\prime} \in W^{n-1, p}\right\}
$$

The spaces $W^{n, p}$ can be characterized as follows.
Theorem 1. Let $n \in \mathbb{N}$. Then, $u \in W^{n, p}$ if and only if there exist functions $g_{1}, \ldots, g_{n} \in L^{p}$ such that

$$
\int_{a}^{b} u(t) \varphi^{(j)}(t) d t=(-1)^{j} \int_{a}^{b} g_{j}(t) \varphi(t) d t
$$

for $\varphi \in C_{c}^{\infty}$ and $j=1, \ldots, n$, where $\varphi^{(j)}$ denotes the classical derivative of $\varphi$ of order $j$. In such a case there exists an absolutely continuous function $\tilde{u}:[a, b] \rightarrow \mathbb{R}^{m}$ such that $u=\tilde{u}$ a.e. on $(a, b)$, which has absolutely continuous classical derivatives $u^{(1)}, \ldots, u^{(n-1)}$, the derivative $u^{(n)}=\left(u^{(n-1)}\right)^{(1)} \in$ $L^{p}$, and

$$
g_{1}=u^{(1)}, \ldots, g_{n}=u^{(n)} .
$$

Remark 2. One shows that $g_{1}=u^{\prime}, g_{2}=\left(u^{\prime}\right)^{\prime}, \ldots, g_{n}=$ $\left(\cdots\left(u^{\prime}\right)^{\prime} \cdots\right)^{\prime}$ ( $n$ times).

Remark 3. In our paper, we will identify functions defined on $(a, b)$ and $[a, b]((a, b],[a, b)$, resp.) that are equal a.e. on $(a, b)$.

Each of the functions $g_{j}, j=1, \ldots, n$, is denoted by $D^{j} u$ and called the weak derivative of $u$ of order $j$.

The space $W^{n, p}$ endowed with a norm

$$
\|u\|_{W^{n, p}}^{p}=\sum_{i=0}^{n}\left\|D^{i} u\right\|_{L^{p}}^{p}, \quad u \in W^{n, p},
$$

where $D^{0} u=u$, has many useful properties such as completeness, reflexivity (for $1<p<\infty$ ), and separability. 
Moreover, some imbeddings of these spaces are compact (cf. [1]).

In the last years, many papers and books on fractional calculus and its applications have appeared. Most of them concern fractional differential equations, including calculus of variations and optimal control. In the classical (positive integer) case the fundamental role in this field is played by the mentioned Sobolev spaces. To our best knowledge, there are no "fractional" Sobolev spaces based on the notion of fractional derivative in Riemann-Liouville sense, which seems to be the most used in the theory of fractional differential equations. Our aim is to give some systematic basics for applications of fractional calculus to differential equations. More precisely, we extend the above definitions, with the aid of the Riemann-Liouville derivatives, to the case of noninteger positive (fractional) order $\alpha$, derive a fractional counterpart of Theorem 1 , and prove the basic properties of the introduced spaces. When $\alpha=n \in \mathbb{N}$, the obtained results reduce to the classical ones. In the literature, some generalizations of Sobolev spaces to noninteger orders, on a domain $\Omega \subset \mathbb{R}^{n}$, are known (cf. [2]): Gagliardo spaces $W^{\alpha, p}(\Omega$ ), Besov spaces $B^{\alpha, p}(\Omega)$, and Nikolskii spaces $H^{\alpha, p}(\Omega)$. They have been introduced with the aid of approaches different from ours and their comparison with our spaces (in the case of $\Omega=(a, b))$ is an open problem. Let us point that only Gagliardo spaces coincide with the classical Sobolev spaces when $\alpha=n$.

The paper is organized as follows. In the second section, we recall some basic notions and facts from the fractional calculus including a characterization of functions possessing the left (right) Riemann-Liouville derivatives. In the third section, we derive some special cases of the fractional theorem on the integration by parts. In the fourth section, we define the fractional Sobolev spaces of any order $\alpha>0$ and characterize them. In the fifth section, we derive a fractional counterpart of Theorem 1, define the weak fractional derivatives of order $\alpha>0$, and show that they coincide with the Riemann-Liouville derivatives. In the sixth section, we introduce two norms in the fractional Sobolev spaces and prove their equivalence. In the seventh section, we derive completeness, reflexivity, and separability of the introduced spaces. In the eighth section, we prove compactness of some imbeddings. In the ninth section, we present some applications of the obtained results to fractional boundary value problems using a variational approach.

In the paper, we limit ourselves to the left fractional Sobolev spaces, but in an analogous way one can define right spaces and derive their appropriate properties.

\section{Preliminaries}

By $A C^{n, 1}=A C^{n, 1}\left([a, b], \mathbb{R}^{m}\right)$, where $n \in \mathbb{N}$, we denote the set of all functions $f:[a, b] \rightarrow \mathbb{R}^{m}$ that have a representative (a.e. on $[a, b]$ ) which is absolutely continuous together with its classical derivatives of orders $1, \ldots, n-1$. Of course, such a function possesses also the classical derivative of order $n$, existing a.e. on $[a, b]$ and belonging to $L^{1}$. These classical derivatives are the weak derivatives $D^{1} f, \ldots, D^{n} f$ of $f$. It is known (cf. [3, Lemma 2.4]) that $f \in A C^{n, 1}$ if and only if there exist $c_{0}, c_{1}, \ldots, c_{n-1} \in \mathbb{R}^{m}$ and $\varphi \in L^{1}$ such that

$$
\begin{aligned}
f(t)= & \sum_{i=0}^{n-1} \frac{c_{i}}{i !}(t-a)^{i} \\
& +\int_{a}^{t} \int_{a}^{t_{1}} \cdots \int_{a}^{t_{n-1}} \varphi(\tau) d \tau d t_{n-1} \cdots d t_{1}, \quad t \in[a, b] \text { a.e. }
\end{aligned}
$$

In such a case,

$$
\begin{gathered}
D^{i} f(a)=c_{i}, \quad i=0, \ldots, n-1, \\
D^{n} f(t)=\varphi(t), \quad t \in[a, b] \text { a.e. }
\end{gathered}
$$

By $A C^{n, p}=A C^{n, p}\left([a, b], \mathbb{R}^{m}\right)$ we denote the set of all functions $f:[a, b] \rightarrow \mathbb{R}^{m}$ that have representation (6) with $c_{0}, c_{1}, \ldots, c_{n-1} \in \mathbb{R}^{m}$ and $\varphi \in L^{p}$. It is known that $A C^{n, p}=W^{n, p}$ for $1 \leq p<\infty$.

Let $\alpha>0$, and let $h \in L^{1}$. By the left Riemann-Liouville fractional integral of $h$ on the interval $[a, b]$ we mean (cf. [3]) a function $I_{a+}^{\alpha} h$ given by

$$
\left(I_{a+}^{\alpha} h\right)(t)=\frac{1}{\Gamma(\alpha)} \int_{a}^{t} \frac{h(\tau)}{(t-\tau)^{1-\alpha}} d \tau, \quad t \in[a, b] \text { a.e. }
$$

Remark 4. The above integral exists and is finite a.e. on $[a, b]$.

In view of the convergence (cf. [3, Theorem 2.7])

$$
\lim _{\alpha \rightarrow 0^{+}}\left(I_{a+}^{\alpha} h\right)(t)=h(t), \quad t \in[a, b] \text { a.e., }
$$

it is natural to put

$$
\left(I_{a+}^{0} h\right)(t)=h(t), \quad t \in[a, b] \text { a.e. }
$$

Remark 5. If $\alpha \geq 1$, the right side of (8) exists (and is finite) everywhere on $[a, b]$. So, $I_{a+}^{\alpha} h$ can be defined everywhere on $[a, b]$. In such a case it is continuous on $[a, b]$.

Remark 6. It is easy to see that if $h$ is essentially bounded on $[a, b]$ and $0<\alpha<1$, then the right side of (8) is defined and bounded everywhere on $(a, b]$. So, in this case $I_{a+}^{\alpha} h$ can be defined everywhere on $(a, b]$. From [3, Theorem 3.6] it follows that in such a case $I_{a+}^{\alpha} h$ is equal everywhere on $(a, b]$ to a continuous function on $[a, b]$. It is also known (cf. [3, Theorem 2.6]) that if $h \in L^{p}$ with $1 \leq p<\infty$, then $I_{a+}^{\alpha} h \in L^{p}$.

Let $n-1<\alpha \leq n$, where $n \in \mathbb{N}$. By $A C_{a+}^{\alpha, 1}=$ $A C_{a+}^{\alpha, 1}\left([a, b], \mathbb{R}^{m}\right)$ we denote the set of all functions $f$ : $[a, b] \rightarrow \mathbb{R}^{m}$ that have the representation

$$
\begin{aligned}
f(t)= & \sum_{i=0}^{n-1} \frac{c_{i}}{\Gamma(\alpha-n+1+i)}(t-a)^{\alpha-n+i} \\
& +I_{a+}^{\alpha} \varphi(t), \quad t \in[a, b] \quad \text { a.e., }
\end{aligned}
$$

with $c_{0}, c_{1}, \ldots, c_{n-1} \in \mathbb{R}^{m}$ and $\varphi \in L^{1}$. Of course, $A C_{a+}^{n, 1}=$ $A C^{n, 1}$. 
We say that $f \in L^{1}$ possesses the left Riemann-Liouville derivative $D_{a+}^{\alpha} f$ of order $\alpha \in(n-1, n], n \in \mathbb{N}$, on the interval $[a, b]$ if $I_{a+}^{n-\alpha} f \in A C^{n, 1}$. By this derivative we mean the derivative $D^{n}\left(I_{a+}^{n-\alpha} f\right)$. Of course, $D_{a+}^{n} f=D^{n} f$.

The next theorem can be deduced from [3, Corollary 2.1, Lemma 2.5 (b), Lemma 2.6 (b)] but, to our best knowledge, it has not been formulated by other authors. In [4] we give a direct proof of it in the case of $n=1$, in [5]-when $n \geq 2$. In the case of $\alpha=n$ it reduces to the theorem on the integral representation of type (6) of functions belonging to $A C^{n, 1}$.

Theorem 7. If $n-1<\alpha \leq n, n \in \mathbb{N}$, and $f \in L^{1}$, then $f$ has the left Riemann-Liouville derivative $D_{a^{+}}^{\alpha} f$ of order $\alpha$ on the interval $[a, b]$ if and only if $f \in A C_{a+}^{\alpha, 1}$; that is, $f$ has the representation (11). In such a case,

$$
\begin{gathered}
D^{i}\left(I_{a+}^{n-\alpha} f\right)(a)=c_{i}, \quad i-0, \ldots, n-1, \\
\left(D_{a+}^{\alpha} f\right)(t)=\varphi(t), \quad t \in[a, b] \text { a.e. }
\end{gathered}
$$

By $A C_{a+}^{\alpha, p}(1 \leq p<\infty)$ we denote the set of all functions $f:[a, b] \rightarrow \mathbb{R}^{m}$ possessing representation (11) with $c_{0}, c_{1}, \ldots, c_{n-1} \in \mathbb{R}^{m}, \varphi \in L^{p}$.

Remark 8. It is easy to see that the above theorem implies the following one (for any $1 \leq p<\infty$ ): $f$ has the left RiemannLiouville derivative $D_{a+}^{\alpha} f \in L^{p}$ if and only if $f \in A C_{a+}^{\alpha, p}$; that is, $f$ has the representation (11) with $\varphi \in L^{p}$.

Let $\alpha>0$. By the right Riemann-Liouville fractional integral of $h \in L^{1}$ on the interval $[a, b]$ we mean a function

$$
\left(I_{b-}^{\alpha} h\right)(t)=\frac{1}{\Gamma(\alpha)} \int_{t}^{b} \frac{h(\tau)}{(\tau-t)^{1-\alpha}} d \tau, \quad t \in[a, b] \text { a.e. }
$$

Similarly, as in the case of left integral, we put

$$
\left(I_{b-}^{0} h\right)(t)=h(t), \quad t \in[a, b] \text { a.e. }
$$

Remark 9. Clearly, $I_{b-}^{\alpha} \varphi$ has the properties analogous to those described in Remarks 5 and 6.

By $A C_{b-}^{\alpha, 1}=A C_{b-}^{\alpha, 1}\left([a, b], \mathbb{R}^{m}\right)$ we denote the set of all functions $f:[a, b] \rightarrow \mathbb{R}^{m}$ that have the representation

$$
\begin{aligned}
f(t)= & \sum_{i=0}^{n-1}(-1)^{i} \frac{d_{i}}{\Gamma(\alpha-n+1+i)}(b-t)^{\alpha-n+i} \\
& +I_{b-}^{\alpha} \psi(\tau), \quad t \in[a, b] \text { a.e., }
\end{aligned}
$$

with $d_{0}, d_{1}, \ldots, d_{n-1} \in \mathbb{R}^{m}$ and $\psi \in L^{1}$. It is easy to see that $A C_{b-}^{n, 1}=A C^{n, 1}$.

We say that $f \in L^{1}$ possesses the right Riemann-Liouville derivative $D_{b-}^{\alpha} f$ of order $\alpha \in(n-1, n], n \in \mathbb{N}$, on the interval $[a, b]$ if $I_{b-}^{n-\alpha} f \in A C^{n, 1}$. By this derivative we mean the function $(-1)^{n} D^{n}\left(I_{b-}^{1-\alpha} f\right)$. Of course, $D_{b-}^{n} f=(-1)^{n} D^{n} f$.

We also have the following.
Theorem 10. If $n-1<\alpha \leq n, n \in \mathbb{N}$, and $f \in L^{1}$, then $f$ has the right Riemann-Liouville derivative $D_{b-}^{\alpha} f$ of order $\alpha$ on the interval $[a, b]$ if and only if $f \in A C_{b-}^{\alpha, 1} f$; that is, $f$ has the representation (15). In such a case,

$$
\begin{gathered}
D^{i}\left(I_{b-}^{n-\alpha} f\right)(b)=d_{i}, \quad i=0, \ldots, n-1, \\
\left(D_{b-}^{\alpha} f\right)(t)=\psi(t), \quad t \in[a, b] \text { a.e. }
\end{gathered}
$$

By $A C_{b-}^{\alpha, p}, 1<p<\infty$, we denote the set of all functions $f:[a, b] \rightarrow \mathbb{R}^{m}$ possessing representation (15) with $d_{0}, d_{1}, \ldots, d_{n-1} \in \mathbb{R}^{m}, \psi \in L^{p}$.

Remark 11. As in the case of left Riemann-Liouville derivative, the following theorem holds true for any $1 \leq p<\infty$ : $f$ has the right Riemann-Liouville derivative $D_{b-}^{\alpha} f \in L^{p}$ if and only if $f \in A C_{b-}^{\alpha, p}$; that is, $f$ has the representation (15) with $\psi \in L^{p}$.

In the next section, we will use the following two theorems (cf. $[6,7])$.

Theorem 12. If $\alpha, \beta>0$, then

$$
A C_{a+}^{\alpha, 1} \subset A C_{a+}^{\beta, 1}
$$

if and only if

$$
\beta \in(0,1], \quad \alpha \in \bigcup_{i=0}^{\infty}[\beta+i, 1+i]
$$

or

$$
\beta \in(1, \infty), \quad \alpha=\beta+i, \quad i=0,1, \ldots .
$$

Theorem 13. (a) If $n-1<\alpha \leq n, n \in \mathbb{N}, 0<\beta<\alpha-n+1$, and $x \in A C_{a+}^{\alpha, 1}$ is of the form (11), then

$$
x(t)=I_{a+}^{\beta} \psi(t), \quad t \in[a, b] \text { a.e. },
$$

where $\psi \in L^{1}$ is given by

$$
\begin{aligned}
\psi(t)= & \sum_{i=0}^{n-1} \frac{c_{i}}{\Gamma(\alpha-n+1-\beta+i)}(t-a)^{\alpha-n-\beta+i} \\
& +I_{a+}^{\alpha-\beta} \varphi(t), \quad t \in[a, b] \text { a.e. }
\end{aligned}
$$

(b) If $n-1<\alpha \leq n, n \in \mathbb{N}, n \geq 2, \beta=\alpha-j, j \in$ $\{1, \ldots, n-1\}$, and $x \in A C_{a+}^{\alpha, 1}$ are of the form (11), then

$$
\begin{aligned}
x(t)= & \sum_{i=0}^{(n-j)-1} \frac{d_{i}}{\Gamma(\beta-(n-j)+1+i)}(t-a)^{\beta-(n-j)+i} \\
& +I_{a+}^{\beta} \psi(t), \quad t \in[a, b] \text { a.e., }
\end{aligned}
$$

where

$$
\begin{gathered}
d_{i}=c_{i}, \quad i=0, \ldots,(n-j)-1, \\
\psi(t)=\sum_{i=0}^{j-1} I_{a+}^{i} c_{n-j+i}(t)+I_{a+}^{j} \varphi(t), \quad t \in[a, b] \text { a.e. }
\end{gathered}
$$




\section{Some Special Cases of Integration by Parts}

Below, $C=C\left([a, b], \mathbb{R}^{m}\right)$ is the set of continuous functions $f$ : $[a, b] \rightarrow \mathbb{R}^{m}$ and $C^{n}=C^{n}\left([a, b], \mathbb{R}^{m}\right)$ is the set of functions $f:[a, b] \rightarrow \mathbb{R}^{m}$ such that $f^{(i)} \in C, i=0, \ldots, n$ (by $f^{(0)}$ we mean the function $f$ ). Of course, these classical derivatives coincide with the weak ones $D^{0} f, D^{1} f, \ldots, D^{n} f$.

Lemma 14. If $n-1<\alpha<n, n \in \mathbb{N}, f \in C^{n}$, and

$$
D^{i} f(b)=0, \quad i=0, \ldots, n-1,
$$

then $I_{b-}^{n-\alpha} f \in C^{n}$,

$$
\begin{gathered}
D^{i}\left(I_{b-}^{n-\alpha} f\right)(b)=0, \quad i=0, \ldots, n-1, \\
D_{b-}^{\alpha} f=I_{b-}^{n-\alpha}\left(D_{b-}^{n} f\right)=I_{b-}^{n-\alpha}\left((-1)^{n} D^{n} f\right) \in C .
\end{gathered}
$$

Consequently, $f \in I_{b-}^{\alpha}(C)$.

Proof. If $f \in C^{n}$ and

$$
D^{i} f(b)=0, \quad i=0, \ldots, n-1,
$$

then (cf. Remark 6 and [3, formulas (2.21) and (2.58)])

$$
\begin{aligned}
I_{b-}^{n-\alpha} f(t) & =I_{b-}^{n-\alpha} I_{b-}^{n}\left(D_{b-}^{n} f\right)(t) \\
& =I_{b-}^{n} I_{b-}^{n-\alpha}\left(D_{b-}^{n} f\right)(t) \\
& =I_{b-}^{n} I_{b-}^{n-\alpha}\left((-1)^{n} D^{n} f\right)(t)
\end{aligned}
$$

for all $t \in[a, b]$. This means (cf. Remark 6) that $I_{b-}^{n-\alpha} f \in C^{n}$ and $D_{b-}^{\alpha} f=I_{b-}^{n-\alpha}\left((-1)^{n} D^{n} f\right) \in C$. Equalities (28) imply also the equalities

$$
D^{i}\left(I_{b-}^{n-\alpha} f\right)(b)=0, \quad i=0, \ldots, n-1 .
$$

So (cf. Theorem 7), $f \in I_{b-}^{\alpha}(C)$.

Remark 15. Analogous lemma can be obtained for the left integral $I_{a+}^{n-\alpha} f$ and derivative $D_{a+}^{\alpha} f$ (without the term $(-1)^{n}$ ).

We have the following special case of the theorem on integration by parts.

Theorem 16. If $n-1<\alpha \leq n, n \in \mathbb{N}$, then

$$
\int_{a}^{b} f(t)\left(D_{b-}^{\alpha} g\right)(t) d t=\int_{a}^{b}\left(D_{a+}^{\alpha} f\right)(t) g(t) d t
$$

for any $f \in A C_{a+}^{\alpha, 1}$ and $g \in C^{n}$ satisfying boundary conditions

$$
D^{i} g(a)=D^{i} g(b)=0, \quad i=0, \ldots, n-1 .
$$

Proof. Let $f \in A C_{a+}^{\alpha, 1}$ be of the form (11) with $c_{0}, c_{1}, \ldots, c_{n-1} \in$ $\mathbb{R}^{m}$ and $\varphi \in L^{1}$. We have (Lemma 14 and Remark 9)

$$
\begin{aligned}
\int_{a}^{b} f( & t)\left(D_{b-}^{\alpha} g\right)(t) d t \\
= & \int_{a}^{b} f(t)\left(I_{b-}^{n-\alpha}\left(D_{b-}^{n} g\right)\right)(t) d t \\
= & \int_{a}^{b}\left(\sum_{i=0}^{n-1} \frac{c_{i}}{\Gamma(\alpha-n+1+i)}(t-a)^{\alpha-n+i}+I_{a+}^{\alpha} \varphi(\tau)\right) \\
= & \sum_{i=0}^{n-1} c_{i} I_{b-}^{\alpha-n+1+i}\left(I_{b-}^{n-\alpha} D_{b-}^{n} g\right)(a) \\
& \left.+\int_{a-}^{n-\alpha}\left(D_{b-}^{n} g\right)\right)(t) d t \\
= & \sum_{i=0}^{n-1} c_{i} I_{b-}^{1+i} D_{b-}^{n} g(a) \\
& \left.+\int_{a+}^{b} f\right)(t)\left(I_{b-}^{n} D_{b-}^{n} g\right)(t) d t \\
= & \int_{a}^{b}\left(D_{a+}^{\alpha} f\right)(t) g(t) d t
\end{aligned}
$$

Existence of the first integral and the first equality follows from Lemma 14; in the third equality we used an integral form of a classical fractional theorem on the integration by parts (cf. [3, formula (2.20)]), in the fourth equality we used continuity of $D^{n} g$ and in the fifth equality we used the equalities

$$
\begin{aligned}
I_{b-}^{1}\left(D_{b-}^{n} g\right)(t) & =\int_{t}^{b}\left(D_{b-}^{n} g\right)(t) d t \\
& =(-1)^{n} \int_{t}^{b}\left(D^{n} g\right)(t) d t \\
& =(-1)^{n}\left(D^{n-1} g(b)-D^{n-1} g(t)\right) \\
& =(-1)^{n-1} D^{n-1} g(t) \\
& =D_{b-}^{n-1} g(t) .
\end{aligned}
$$

The proof is completed.

The next theorem will be used in the last section of the paper. Proof of this theorem is contained in [4] and in [5] its extension to the case of fractional derivatives of higher order is derived (the method of the proof is the same in both papers). 
Theorem 17. If $0<\alpha<1, \alpha>1 / p, \alpha>1 / q, 1 \leq p<\infty$, and $1 \leq q<\infty$, then

$$
\begin{aligned}
\int_{a}^{b} f(t)\left(D_{b-}^{\alpha} g\right)(t) d t= & \left(I_{a+}^{1-\alpha} f\right)(a) g(a) \\
& -\left(I_{b-}^{1-\alpha} g\right)(b) f(b) \\
& +\int_{a}^{b}\left(D_{a+}^{\alpha} f\right)(t) g(t) d t
\end{aligned}
$$

for $f \in A C_{a+}^{\alpha, p}\left([a, b], \mathbb{R}^{n}\right)$ and $g \in A C_{b-}^{\alpha, q}\left([a, b], \mathbb{R}^{n}\right)$.

\section{Fractional Sobolev Spaces}

Let $0<\alpha \leq 1$ and let $1 \leq p<\infty$. By left Sobolev space of order $\alpha$ we will mean the set $W_{a+}^{\alpha, p}=W_{a+}^{\alpha, p}\left((a, b), \mathbb{R}^{m}\right)$ given by

$$
\begin{gathered}
W_{a+}^{\alpha, p}=\{u \\
\in L^{p} ; \underset{g \in L^{p} \varphi \in C_{c}^{\infty}}{\forall} \int_{a}^{b} u(t) D_{b-}^{\alpha} \varphi(t) d t \\
\left.=\int_{a}^{b} g(t) \varphi(t) d t\right\} .
\end{gathered}
$$

A function $g$ given above will be called the weak left fractional derivative of order $\alpha \in(0,1]$ of $u$; let us denote it by $u_{a+}^{\alpha}$. Uniqueness of this weak derivative follows from [1, Lemma IV.2 and Propositions IV.18, IV.21].

Let $n-1<\alpha \leq n, n \in \mathbb{N}, n \geq 2$. By the left Sobolev space of order $\alpha$ we mean the set $W_{a+}^{\alpha, p}=W_{a+}^{\alpha, p}\left([a, b], \mathbb{R}^{m}\right)$ given by

$$
W_{a+}^{\alpha, p}=\left\{u \in W_{a+}^{\alpha-1, p} ; u_{a+}^{\alpha-(n-1)} \in W_{a+}^{n-1, p}\right\} .
$$

Remark 18. Since $D_{b-}^{1} \varphi=-D^{1} \varphi=-\varphi^{(1)}$ for $\varphi \in C_{c}^{\infty}$, we see that the weak left fractional derivative $u_{a+}^{1}$ of $u$ coincides with the classical weak derivative $u^{\prime}=D^{1} u$ of $u$. Consequently,

$$
W_{a+}^{n, p}=W^{n, p}=A C^{n, p}=A C_{a+}^{n, p}
$$

for $n \in \mathbb{N}$.

We have the following characterization of $W_{a+}^{\alpha, p}$.

Theorem 19. If $n-1<\alpha \leq n, n \in \mathbb{N}$, and $1 \leq p<\infty$, then

$$
W_{a+}^{\alpha, p}=A C_{a+}^{\alpha, p} \cap L^{p} .
$$

Proof. Case of $\alpha=n$ follows from (37) and from the fact that $A C_{a+}^{n, p} \subset L^{p}$. So, let us consider the case of $n-1<\alpha<n$. We will apply the induction with respect to $n \in \mathbb{N}$.

Let $n=1$. If $u \in A C_{a+}^{\alpha, p} \cap L^{p}$, then from Theorem 7 it follows that $u$ has the derivative $D_{a+}^{\alpha} u \in L^{p}$. Theorem 16 implies that

$$
\int_{a}^{b} u(t) D_{b-}^{\alpha} \varphi(t) d t=\int_{a}^{b}\left(D_{a+}^{\alpha} u\right)(t) \varphi(t) d t
$$

for any $\varphi \in C_{c}^{\infty}$. So, $u \in W_{a+}^{\alpha, p}$ with

$$
u_{a+}^{\alpha}=g=D_{a+}^{\alpha} u \in L^{p} .
$$

Now, let us assume that $u \in W_{a+}^{\alpha, p}$, that is, $u \in L^{p}$, and there exists a function $g \in L^{p}$ such that

$$
\int_{a}^{b} u(t) D_{b-}^{\alpha} \varphi(t) d t=\int_{a}^{b} g(t) \varphi(t) d t
$$

for any $\varphi \in C_{c}^{\infty}$. To show that $u \in A C_{a+}^{\alpha, p} \cap L^{p}$ it is sufficient to check (cf. Theorem 7 and definition of $A C_{a+}^{\alpha, p}$ ) that $u$ possesses the left Riemann-Liouville derivative of order $\alpha$, belonging to $L^{p}$, that is, that $I_{a+}^{1-\alpha} u$ is absolutely continuous on $[a, b]$ and its classical derivative of the first order (existing a.e. on $[a, b]$ ) belongs to $L^{p}$.

If $\varphi \in C_{c}^{\infty}$, then (cf. Lemma 14) $\varphi \in I_{b-}^{\alpha}(C)$ and $D_{b-}^{\alpha} \varphi=I_{b-}^{1-\alpha}\left(-D^{1} \varphi\right)$. From the differential form of the classical fractional theorem on the integration by parts (cf. [3, formula (2.64)]) it follows that

$$
\begin{aligned}
\int_{a}^{b} u( & (t) D_{b-}^{\alpha} \varphi(t) d t \\
& =\int_{a}^{b} u(t)\left(I_{b-}^{1-\alpha}\left(-D^{1} \varphi\right)\right)(t) d t \\
& =\int_{a}^{b}\left(D_{a+}^{1-\alpha} I_{a+}^{1-\alpha} u\right)(t)\left(I_{b-}^{1-\alpha}\left(-D^{1} \varphi\right)\right)(t) d t \\
& =\int_{a}^{b}\left(I_{a+}^{1-\alpha} u\right)(t)\left(-D^{1} \varphi\right)(t) d t \\
& =-\int_{a}^{b}\left(I_{a+}^{1-\alpha} u\right)(t)\left(D^{1} \varphi\right)(t) d t .
\end{aligned}
$$

This means (cf. (41) and (42)) that

$$
\int_{a}^{b}\left(I_{a+}^{1-\alpha} u\right)(t)\left(D^{1} \varphi\right)(t) d t=-\int_{a}^{b} g(t) \varphi(t) d t
$$

for any $\varphi \in C_{c}^{\infty}$. So, $I_{a+}^{1-\alpha} u \in W^{1, p}$. Consequently, $I_{a+}^{1-\alpha} u$ is absolutely continuous and its classical derivative is equal a.e. on $[a, b]$ to $g \in L^{p}$.

Now, let $n \in \mathbb{N}$ and assume that

$$
W_{a+}^{\alpha, p}=A C_{a+}^{\alpha, p} \cap L^{p}
$$

for any $\alpha \in(n-1, n)$. We will show that this equality holds true for any $\alpha \in(n, n+1)$.

Indeed, let $\alpha \in(n, n+1)$. If $u \in W_{a+}^{\alpha, p}$, then $u \in W_{a+}^{\alpha-1, p}=$ $A C_{a+}^{\alpha-1, p} \cap L^{p}$ and $u_{a+}^{\alpha-n} \in W_{a+}^{n, p}$. So,

$$
\begin{aligned}
u(t)= & \sum_{i=0}^{n-1} \frac{c_{i}}{\Gamma(\alpha-n+i)}(t-a)^{\alpha-1-n+i} \\
& +I_{a+}^{\alpha-1} \varphi(t), \quad t \in[a, b] \text { a.e., }
\end{aligned}
$$

where $c_{0}, c_{1}, \ldots, c_{n-1} \in \mathbb{R}^{m}$ and $\varphi \in L^{p}$. If $n=1$, from the above formula it follows (cf. (40)) that

$$
\varphi=D_{a+}^{\alpha-1} u=u_{a+}^{\alpha-1} \in W_{a+}^{1, p}=W^{1, p} .
$$


If $n \geq 2$, from Theorem 13 (b) and (40) it follows that

$$
\begin{aligned}
u_{a+}^{\alpha-n} & =D_{a+}^{\alpha-n} u=D_{a+}^{(\alpha-1)-(n-1)} u \\
& =\sum_{i=0}^{n-2} I_{a+}^{i} c_{1+i}+I_{a+}^{n-1} \varphi .
\end{aligned}
$$

This means that $I_{a+}^{n-1} \varphi \in W_{a+}^{n, p}$ and, consequently,

$$
\begin{aligned}
\varphi & =D_{a+}^{n-1} I_{a+}^{n-1} \varphi=D^{n-1} I_{a+}^{n-1} \varphi \in D^{n-1}\left(W_{a+}^{n, p}\right) \\
& =D^{n-1}\left(W^{n, p}\right)=W^{1, p} .
\end{aligned}
$$

So, in both cases $(n=1, n \geq 2)$ there exist $c_{n} \in \mathbb{R}^{m}$ and $\lambda \in L^{p}$ such that

$$
\varphi=c_{n}+I_{a+}^{1} \lambda
$$

To show that $u \in A C_{a+}^{\alpha, p} \cap L^{p}$ it is sufficient to check that

$$
I_{a+}^{n+1-\alpha} u \in A C_{a+}^{n+1, p} .
$$

Indeed, we have (below, we use the following elementary formula

$$
\begin{array}{r}
I_{a+}^{\nu}\left((\cdot-a)^{\delta-1}\right)(t)=\frac{\Gamma(\delta)}{\Gamma(\nu+\delta)}(t-a)^{\nu+\delta-1} \\
t \in[a, b] \text { a.e., }
\end{array}
$$

for $v>0, \delta>0)$

$$
\begin{aligned}
I_{a+}^{n+1-\alpha} u(t)= & \sum_{i=0}^{n-1} \frac{c_{i}}{\Gamma(\alpha-n+i)} I_{a+}^{n+1-\alpha}\left((\cdot-a)^{\alpha-1-n+i}\right)(t) \\
& +I_{a+}^{n+1-\alpha} I_{a+}^{\alpha-1} \varphi(t) \\
= & \sum_{i=0}^{n-1} \frac{c_{i}}{\Gamma(\alpha-n+i)} \frac{\Gamma(\alpha-n+i)}{\Gamma(1+i)}(t-a)^{i} \\
& +I_{a+}^{n} \varphi(t) \\
= & \sum_{i=0}^{n-1} \frac{c_{i}}{\Gamma(1+i)}+I_{a+}^{n} c_{n}(t)+I_{a+}^{n+1} \lambda(t) \\
= & \sum_{i=0}^{n} \frac{c_{i}}{i !}(t-a)^{i}+I_{a+}^{n+1} \lambda(t)
\end{aligned}
$$

for $t \in[a, b]$ a.e. Thus, $I_{a+}^{n+1-\alpha} u \in A C_{a+}^{n+1, p}$.

Conversely, let $u \in A C_{a+}^{\alpha, p} \cap L^{p}$. To show that $u \in W_{a+}^{\alpha, p}$ we have to check that

$$
u \in W_{a+}^{\alpha-1, p}, \quad u_{a+}^{\alpha-n} \in W_{a+}^{n, p} .
$$

Theorem 13(b) implies the equality

$$
D_{a+}^{\alpha-1} u=c_{n}+I_{a+}^{1} \varphi,
$$

where

$$
\begin{aligned}
u(t)= & \sum_{i=0}^{n} \frac{c_{i}}{\Gamma(\alpha-n+i)}(t-a)^{\alpha-(n+1)+i} \\
& +I_{a+}^{\alpha} \varphi(t), \quad t \in[a, b] \text { a.e., }
\end{aligned}
$$

with $\varphi \in L^{p}$. So (cf. Theorem 12), $u \in A C_{a+}^{\alpha-1, p} \cap L^{p}=W_{a+}^{\alpha-1, p}$. From Theorem 13 (b) it also follows that

$$
\begin{aligned}
u_{a+}^{\alpha-n} & =D_{a+}^{\alpha-n} u=\sum_{i=0}^{n-1} I_{a+}^{i} c_{1+i}+I_{a+}^{n} \varphi \\
& =\sum_{i=0}^{n-1} \frac{c_{1+i}}{i !}(t-a)^{i}+I_{a+}^{n} \varphi \in A C_{a+}^{n, p} \\
& =W_{a+}^{n, p}
\end{aligned}
$$

(existence of the weak fractional derivative $u_{a+}^{\alpha-n}$ and equality $u_{a+}^{\alpha-n}=D_{a+}^{\alpha-n} u$ follow from the relation $u \in W_{a+}^{\alpha-n, p}$ and (40)).

From the first part of the above proof (case of $n=1$ ) and from the uniqueness of the weak fractional derivative the following theorem follows (cf. also [4, fractional fundamental lemma]).

Theorem 20. If $\alpha \in(0,1]$, then the weak left fractional derivative $u_{a+}^{\alpha}$ of a function $u \in W_{a+}^{\alpha, p}$ coincides with its left Riemann-Liouville fractional derivative $D_{a+}^{\alpha}$ u a.e. on $[a, b]$.

Remark 21. If $n-1<\alpha \leq n$ and $(n-\alpha) p<1$, then $A C_{a+}^{\alpha, p} \subset L^{p}$ and, consequently, $W_{a+}^{\alpha, p}=A C_{a+}^{\alpha, p} \cap L^{p}=A C_{a+}^{\alpha, p}$. If $(n-\alpha) p \geq 1$, then $W_{a+}^{\alpha, p}=A C_{a+}^{\alpha, p} \cap L^{p}$ is the set of all functions belonging to $A C_{a+}^{\alpha, p}$ that satisfy the condition $\left(I_{a+}^{n-\alpha} f\right)(a)=0$.

\section{Weak Fractional Derivatives}

Now, we will prove an extension of Theorem 1.

Theorem 22. Let $n-1<\alpha \leq n, n \in \mathbb{N}, 1 \leq p<\infty$, and $u \in L^{p}$. Then, $u \in W_{a+}^{\alpha, p}$ if and only if there exist functions $g_{1}, \ldots, g_{n} \in L^{p}$ such that

$$
\int_{a}^{b} u(t) D_{b-}^{\alpha-(n-i)} \varphi(t) d t=\int_{a}^{b} g_{i}(t) \varphi(t) d t, \quad \varphi \in C_{c}^{\infty},
$$

for any $i \in\{1, \ldots, n\}$. In such a case there exist the left Riemann-Liouville derivatives $D_{a+}^{\alpha-(n-1)} u, D_{a+}^{\alpha-(n-2)} u, \ldots, D_{a+}^{\alpha} u$ of $u$ and

$$
g_{1}=D_{a+}^{\alpha-(n-1)} u, \quad g_{2}=D_{a+}^{\alpha-(n-2)} u, \ldots, \quad g_{n}=D_{a+}^{\alpha} u .
$$

Proof. Case of $\alpha=n$ follows from Theorem 1. So, let us consider the case of $n-1<\alpha<n$. We will apply the induction with respect to $n \in \mathbb{N}$. 
When $n=1$, it is sufficient to use the definition of $W_{a+}^{\alpha, p}$ with $\alpha \in(0,1)$ and Theorem 20 .

So, let $n \in \mathbb{N}$ and assume that the theorem is true for any $\alpha \in(n-1, n)$. We will show that it is true for any $\alpha \in(n, n+1)$.

Indeed, if $u \in W_{a+}^{\alpha, p}$ with a fixed $\alpha \in(n, n+1)$, then

$$
\begin{gathered}
u \in W_{a+}^{\alpha-1, p}, \\
D_{a+}^{\alpha-n} u=u_{a+}^{\alpha-n} \in W_{a+}^{n, p} .
\end{gathered}
$$

Relation (59) implies (by the induction assumption) the existence of functions $g_{1}, \ldots, g_{n} \in L^{p}$ such that (of course, $\alpha-1 \in(n-1, n))$

$$
\begin{aligned}
\int_{a}^{b} u(t) D_{b-}^{\alpha-((n+1)-i)} \varphi(t) d t & =\int_{a}^{b} u(t) D_{b-}^{(\alpha-1)-(n-i)} \varphi(t) d t \\
& =\int_{a}^{b} g_{i}(t) \varphi(t) d t
\end{aligned}
$$

for any $\varphi \in C_{c}^{\infty}$ and $i \in\{1, \ldots, n\}$. Moreover,

$$
\begin{gathered}
g_{1}=D_{a+}^{(\alpha-1)-(n-1)} u=D_{a+}^{\alpha-((n+1)-1)} u, \\
g_{2}=D_{a+}^{(\alpha-1)-(n-2)} u=D_{a+}^{\alpha-((n+1)-2)} u, \\
\vdots \\
g_{n}=D_{a+}^{(\alpha-1)-(n-n)} u=D_{a+}^{\alpha-((n+1)-n)} u .
\end{gathered}
$$

From the existence of $D_{a+}^{\alpha-n} u$ and from (60) it follows that $I_{a+}^{1-(\alpha-n)} u \in W^{n+1, p}$. In particular, there exists derivative $D_{a+}^{\alpha} u \in L^{p}$. If we put $g_{n+1}=D_{a+}^{\alpha} u$ we see that $g_{n+1}=$ $D_{a+}^{\alpha-((n+1)-(n+1))} u$ and

$$
\begin{aligned}
\int_{a}^{b} u(t) D_{b-}^{\alpha-((n+1)-(n+1))} \varphi(t) d t & =\int_{a}^{b} u(t) D_{b-}^{\alpha} \varphi(t) d t \\
& =\int_{a}^{b} D_{a+}^{\alpha} u(t) \varphi(t) d t \\
& =\int_{a}^{b} g_{n+1}(t) \varphi(t) d t
\end{aligned}
$$

for any $\varphi \in C_{c}^{\infty}$ (the second equality follows from Theorem 16).

Now, let us assume that there exist functions $g_{1}, \ldots, g_{n+1} \in L^{p}$ such that

$$
\int_{a}^{b} u(t) D_{b-}^{\alpha-((n+1)-i)} \varphi(t) d t=\int_{a}^{b} g_{i}(t) \varphi(t) d t, \quad \varphi \in C_{c}^{\infty}
$$

for any $i \in\{1, \ldots, n+1\}$. Since $\alpha-((n+1)-i)=\alpha-1-(n-$ $i)$, the above condition for $i \in\{1, \ldots, n\}$ and the induction assumption mean that

$$
u \in W_{a+}^{\alpha-1, p}
$$

Moreover, condition (64) for $i=1$, Lemma 14, and the integral form of the classical fractional theorem on the integration by parts (cf. [3, formula (2.20)]) imply that

$$
\begin{aligned}
\int_{a}^{b} g_{1}(t) \varphi(t) d t & =\int_{a}^{b} u(t) D_{b-}^{\alpha-n} \varphi(t) d t \\
& =\int_{a}^{b} u(t) I_{b-}^{n-\alpha+1} D_{b-}^{1} \varphi(t) d t \\
& =\int_{a}^{b} I_{a+}^{n-\alpha+1} u(t) D_{b-}^{1} \varphi(t) d t \\
& =-\int_{a}^{b} I_{a+}^{n-\alpha+1} u(t) D^{1} \varphi(t) d t
\end{aligned}
$$

for any $\varphi \in C_{c}^{\infty}$. This means that $I_{a+}^{n-\alpha+1} u \in W^{1, p}$. So, if we fix $i \in\{2, \ldots, n+1\}$ and use once again Lemma 14 and the integral form of the classical fractional theorem on the integration by parts, we obtain

$$
\begin{aligned}
\int_{a}^{b} g_{i} & (t) \varphi(t) d t \\
& =\int_{a}^{b} u(t) D_{b-}^{\alpha-((n+1)-i)} \varphi(t) d t \\
& =\int_{a}^{b} u(t) I_{b-}^{(n+1)-\alpha} D_{b-}^{i} \varphi(t) d t \\
& =\int_{a}^{b} I_{a+}^{(n+1)-\alpha} u(t) D_{b-}^{i} \varphi(t) d t \\
& =(-1)^{i} \int_{a}^{b} I_{a+}^{(n+1)-\alpha} u(t) D^{i} \varphi(t) d t \\
& =(-1)^{i} \int_{a}^{b} I_{a+}^{(n+1)-\alpha} u(t) D^{1} D^{i-1} \varphi(t) d t \\
& =(-1)^{i-1} \int_{a}^{b} D^{1} I_{a+}^{1-(\alpha-n)} u(t) D^{i-1} \varphi(t) d t \\
& =(-1)^{i-1} \int_{a}^{b} D_{a+}^{\alpha-n} u(t) D^{i-1} \varphi(t) d t .
\end{aligned}
$$

Thus, from Theorem 1 it follows that

$$
u_{a+}^{\alpha-n}=D_{a+}^{\alpha-n} u \in W^{n, p} .
$$

Using (65) and (68) we assert that $u \in W_{a+}^{\alpha-1, p}$.

Functions $g_{1}, \ldots, g_{n}$ will be called the weak left fractional derivatives of $u \in W_{a+}^{\alpha, p}$ of orders $\alpha-(n-1), \ldots, \alpha$, respectively. Their uniqueness follows from [1, Lemma IV.2 and Propositions IV.18,IV.21]. From the above theorem it follows that they coincide with the appropriate RiemannLiouville derivatives.

We have the following counterpart of Remark 2.

Theorem 23. If $u \in W_{a+}^{\alpha, p}, n-1<\alpha \leq n$, and $n \in \mathbb{N}$, then

$$
g_{i}=D^{i-1}\left(D_{a+}^{\alpha-(n-1)} u\right), \quad i=1, \ldots, n \text {. }
$$


Proof. If $u \in W_{a+}^{\alpha, p}$, then $I_{a+}^{n-\alpha} u \in W^{n, p}$ and from the definition of the Riemann-Liouville derivative and Theorem 22 it follows that

$$
\begin{gathered}
g_{1}=D_{a+}^{\alpha-n+1} u=D^{1} I_{a+}^{1-(\alpha-n+1)} u=D^{1}\left(I_{a+}^{n-\alpha} u\right) \\
g_{2}=D_{a+}^{\alpha-n+2} u=D^{2} I_{a+}^{2-(\alpha-n+2)} u=D^{2} I_{a+}^{n-\alpha} u \\
=D^{1}\left(D^{1} I_{a+}^{n-\alpha} u\right)=D^{1}\left(D_{a+}^{\alpha-n+1} u\right) \\
\vdots \\
g_{n}=D_{a+}^{\alpha-n+n} u=D^{n} I_{a+}^{n-(\alpha-n+n)} u \\
=D^{n-1}\left(D^{1} I_{a+}^{n-\alpha} u\right)=D^{n-1}\left(D_{a+}^{\alpha-n+1} u\right) .
\end{gathered}
$$

The proof is completed.

\section{Norms in $W_{a+}^{\alpha, p}$}

Let us fix $\alpha \in(n-1, n]$ where $n \in \mathbb{N}$ and consider in the space $W_{a+}^{\alpha, p}$ a norm $\|\cdot\|_{W_{a+}^{\alpha, p}}$ given by

$$
\|u\|_{W_{a+}^{\alpha, p}}^{p}=\|u\|_{L^{p}}^{p}+\sum_{i=0}^{n-1}\left\|D_{a+}^{\alpha-(n-1)+i} u\right\|_{L^{p}}^{p}, \quad u \in W_{a+}^{\alpha, p}
$$

(here $\|\cdot\|_{L^{p}}$ denotes the classical norm in $L^{p}$ ).

We have the following theorem.

Theorem 24. If $n \in \mathbb{N}$ and $\alpha \in(n-1, n]$, then the norm $\|\cdot\|_{W_{a+}^{\alpha, p}}$ is equivalent to a norm $\|u\|_{a, W_{a+}^{\alpha, p}}$ given by

$$
\|u\|_{a, W_{a+}^{\alpha, p}}^{p}=\sum_{i=0}^{n-1}\left|D^{i} I_{a+}^{n-\alpha} u(a)\right|^{p}+\left\|D_{a+}^{\alpha} u\right\|_{L^{p}}^{p}, \quad u \in W_{a+}^{\alpha, p}
$$

Proof. We will use the induction with respect to $n \in \mathbb{N}$.

Assume that $n=1$ and $(1-\alpha) p<1$. Then, for $u \in W_{a+}^{\alpha, p}$ given by

$$
u(t)=\frac{c}{\Gamma(\alpha)} \frac{1}{(t-a)^{1-\alpha}}+I_{a+}^{\alpha} \varphi(t)
$$

with $c \in \mathbb{R}^{n}$ and $\varphi \in L^{p}$, we have

$$
\begin{aligned}
\|u\|_{L^{p}}^{p} & =\int_{a}^{b}\left|\frac{c}{\Gamma(\alpha)} \frac{1}{(t-a)^{1-\alpha}}+I_{a+}^{\alpha} \varphi(t)\right|^{p} d t \\
& \leq 2^{p-1}\left(\frac{|c|^{p}}{\Gamma(\alpha)^{p}} \int_{a}^{b}(t-a)^{(\alpha-1) p} d t+\left\|I_{a+}^{\alpha} \varphi\right\|_{L^{p}}^{p}\right) \\
& \leq 2^{p-1}\left(\frac{|c|^{p}}{\Gamma(\alpha)^{p}} \frac{1}{(\alpha-1) p+1}(b-a)^{(\alpha-1) p+1}+K^{p}\|\varphi\|_{L^{p}}^{p}\right),
\end{aligned}
$$

where $K=(b-a)^{\alpha} / \Gamma(\alpha+1)$ (cf. [3, formula (2.72)]). Thus (of course, $c=I_{a+}^{1-\alpha} u(a)$ and $\left.\varphi=D_{a+}^{\alpha} u\right)$,

$$
\begin{aligned}
\|u\|_{L^{p}}^{p} & \leq L_{\alpha, 0}\left(|c|^{p}+\|\varphi\|_{L^{p}}^{p}\right) \\
& =L_{\alpha, 0}\left(\left|I_{a+}^{1-\alpha} u(a)\right|^{p}+\left\|D_{a+}^{\alpha} u\right\|_{L^{p}}^{p}\right) \\
& =L_{\alpha, 0}\|u\|_{a, W_{a+}^{\alpha, p}}^{p}
\end{aligned}
$$

where

$$
L_{\alpha, 0}=2^{p-1}\left(\frac{(b-a)^{1-(1-\alpha) p}}{\Gamma(\alpha)^{p}(1-(1-\alpha) p)}+K^{p}\right) .
$$

Consequently,

$$
\|u\|_{W_{a+}^{\alpha, p}}^{p}=\|u\|_{L^{p}}^{p}+\left\|D_{a+}^{\alpha} u\right\|_{L^{p}}^{p} \leq L_{\alpha, 1}\|u\|_{a, W_{a+}^{\alpha, p}}^{p},
$$

where $L_{\alpha, 1}=L_{\alpha, 0}+1$.

Now, we will prove that there exists a constant $M_{\alpha, 1}>0$ such that

$$
\|u\|_{a, W_{a+}^{\alpha, p}}^{p} \leq M_{\alpha, 1}\|u\|_{W_{a+}^{\alpha, p}}^{p}, \quad u \in W_{a+}^{\alpha, p} .
$$

Indeed, let $u \in W_{a+}^{\alpha, p}$ and consider a coordinate function $\left(I_{a+}^{1-\alpha} u\right)^{i}$ of $I_{a+}^{1-\alpha} u$ with a fixed $i \in\{1, \ldots, m\}$. The mean value theorem implies the existence of $t_{0} \in(a, b)$ such that

$$
\left(I_{a+}^{1-\alpha} u\right)^{i}\left(t_{0}\right)=\frac{1}{b-a} \int_{a}^{b}\left(I_{a+}^{1-\alpha} u\right)^{i}(s) d s .
$$

From the absolute continuity of $\left(I_{a+}^{1-\alpha} u\right)^{i}$ it follows that

$$
\left(I_{a+}^{1-\alpha} u\right)^{i}(t)=\left(I_{a+}^{1-\alpha} u\right)^{i}\left(t_{0}\right)+\int_{t_{0}}^{t} D^{1}\left(I_{a+}^{1-\alpha} u\right)^{i}(s) d s
$$

for any $t \in[a, b]$. Consequently,

$$
\begin{aligned}
\left|\left(I_{a+}^{1-\alpha} u\right)^{i}(t)\right| & \leq \frac{1}{b-a}\left\|I_{a+}^{1-\alpha} u\right\|_{L^{1}}+\int_{t_{0}}^{t}\left|D_{a+}^{\alpha} u(s)\right| d s \\
& \leq \frac{1}{b-a}\left\|I_{a+}^{1-\alpha} u\right\|_{L^{1}}+\left\|D_{a+}^{\alpha} u\right\|_{L^{1}} \\
& \leq \frac{1}{b-a} \frac{(b-a)^{1-\alpha}}{\Gamma(2-\alpha)}\|u\|_{L^{1}}+\left\|D_{a+}^{\alpha} u\right\|_{L^{1}}
\end{aligned}
$$

for $t \in[a, b]$. In particular,

$$
\left|\left(I_{a+}^{1-\alpha} u\right)^{i}(a)\right| \leq \frac{1}{b-a} \frac{(b-a)^{1-\alpha}}{\Gamma(2-\alpha)}\|u\|_{L^{1}}+\left\|D_{a+}^{\alpha} u\right\|_{L^{1}} .
$$

So,

$$
\begin{aligned}
\left|I_{a+}^{1-\alpha} u(a)\right| & \leq m M_{\alpha, 0}\left(\|u\|_{L^{1}}+\left\|D_{a+}^{\alpha} u\right\|_{L^{1}}\right) \\
& \leq m M_{\alpha, 0}(b-a)^{(p-1) / p}\left(\|u\|_{L^{p}}+\left\|D_{a+}^{\alpha} u\right\|_{L^{p}}\right),
\end{aligned}
$$


where $M_{\alpha, 0}=(b-a)^{-\alpha} / \Gamma(2-\alpha)+1$. Thus,

$$
\begin{aligned}
\left|I_{a+}^{1-\alpha} u(a)\right|^{p} \leq & m^{p} M_{\alpha, 0}^{p}(b-a)^{p-1} 2^{p-1} \\
& \times\left(\|u\|_{L^{p}}^{p}+\left\|D_{a+}^{\alpha} u\right\|_{L^{p}}^{p}\right)
\end{aligned}
$$

and, consequently,

$$
\begin{aligned}
\|u\|_{a, W_{a+}^{\alpha, p}}^{p}= & \left|I_{a+}^{1-\alpha} u(a)\right|^{p}+\left\|D_{a+}^{\alpha} u\right\|_{L^{p}}^{p} \\
\leq & \left(m^{p} M_{\alpha, 0}^{p}(b-a)^{p-1} 2^{p-1}+1\right) \\
& \times\left(\|u\|_{L^{p}}^{p}+\left\|D_{a+}^{\alpha} u\right\|_{L^{p}}^{p}\right) \\
= & M_{\alpha, 1}\|u\|_{W_{a+}^{\alpha, p}}^{p},
\end{aligned}
$$

where $M_{\alpha, 1}=m^{p} M_{\alpha, 0}^{p}(b-a)^{p-1} 2^{p-1}+1$.

When $(1-\alpha) p \geq 1$, then (cf. Remark 21) $W_{a+}^{\alpha, p}=A C_{a+}^{\alpha, p} \cap$ $L^{p}$ is the set of all functions $u$ belonging to $A C_{a+}^{\alpha, p}$ that satisfy the condition $\left(I_{a+}^{1-\alpha} u\right)(a)=0$. Consequently, in the same way as in the case of $(1-\alpha) p<1$ (putting $c=0$ ) we obtain the inequality $\|u\|_{W_{\alpha+}^{\alpha, p}}^{p} \leq L_{\alpha, 1}\|u\|_{a, W_{a+}^{\alpha, p}}^{p}$ with some $L_{\alpha, 1}>0$. The inequality $\|u\|_{a, W_{a+}^{\alpha, p}}^{p^{a+}} \leq M_{\alpha, 1}\|u\|_{W_{a+}^{\alpha, p}}^{p}$ with some $M_{\alpha, 1}>0$ is obvious (it is sufficient to put $M_{\alpha, 1}=1$ and use the fact that $\left.\left(I_{a+}^{1-\alpha} u\right)(a)=0\right)$.

Now, let us assume that the assertion holds true for some $n \in \mathbb{N}$. We will prove that it is true for $n+1$.

Let $\alpha \in(n, n+1]$. We know that there exist constants $L_{\alpha, n}$ and $M_{\alpha, n}>0$ such that

$$
\left(\frac{1}{M_{\alpha, n}}\right)\|u\|_{a, W_{a+}^{\alpha-1, p}}^{p} \leq\|u\|_{W_{a+}^{\alpha-1, p}}^{p} \leq L_{\alpha, n}\|u\|_{a, W_{a+}^{\alpha-1, p}}^{p}
$$

for any $u \in W_{a+}^{\alpha-1, p}$. If $u \in W_{a+}^{\alpha, p}$, then $u \in W_{a+}^{\alpha-1, p}$ and therefore (from the induction assumption)

$$
\begin{aligned}
\|u\|_{W_{a+}^{\alpha, p}}^{p}= & \|u\|_{L^{p}}^{p}+\sum_{i=0}^{n}\left\|D_{a+}^{\alpha-n+i} u\right\|_{L^{p}}^{p} \\
= & \|u\|_{L^{p}}^{p}+\sum_{i=0}^{n-1}\left\|D_{a+}^{(\alpha-1)-(n-1)+i} u\right\|_{L^{p}}^{p} \\
& +\left\|D_{a+}^{\alpha} u\right\|_{L^{p}}^{p} \\
= & \|u\|_{W_{a+}^{\alpha-1, p}}^{p}+\left\|D_{a+}^{\alpha} u\right\|_{L^{p}}^{p} \\
\leq & L_{\alpha-1, n}\|u\|_{a, W_{a+}^{p}}^{\alpha-1, p}+\left\|D_{a+}^{\alpha} u\right\|_{L^{p}}^{p} \\
= & L_{\alpha-1, n}\left(\sum_{i=0}^{n-1}\left|D^{i} I_{a+}^{n-(\alpha-1)} u(a)\right|^{p}+\left\|D_{a+}^{\alpha-1} u\right\|_{L^{p}}^{p}\right) \\
& +\left\|D_{a+}^{\alpha} u\right\|_{L^{p} .}^{p}
\end{aligned}
$$

So,

$$
\begin{aligned}
& \sum_{i=0}^{n-1}\left|D^{i} I_{a+}^{n-(\alpha-1)} u(a)\right|^{p} \\
& \quad \leq M_{\alpha-1, n}\left(\|u\|_{L^{p}}^{p}+\sum_{i=0}^{n-2}\left\|D_{a+}^{\alpha-1-(n-1)+i} u\right\|_{L^{p}}^{p}+\left\|D_{a+}^{\alpha-1} u\right\|_{L^{p}}^{p}\right) .
\end{aligned}
$$


Let us recall that $I_{a+}^{n+1-\alpha} u \in W^{n+1, p}$, so $I_{a+}^{n+1-\alpha} u \in W^{n, p}$, $D^{n} I_{a+}^{n+1-\alpha} u \in W^{1, p}$, and $D_{a+}^{\alpha} u=D^{n+1} I_{a+}^{n+1-\alpha} u=D^{1} D^{n} I_{a+}^{n+1-\alpha} u$. Thus,

$$
\begin{aligned}
& \left|D^{n} I_{a+}^{n+1-\alpha} u(a)\right|^{p}+\left\|D_{a+}^{\alpha} u\right\|_{L^{p}}^{p} \\
& \quad=\left\|D^{n} I_{a+}^{n+1-\alpha} u\right\|_{a, W_{a+}^{1, p}}^{p} \\
& \quad \leq M_{1,1}\left\|D^{n}\left(I_{a+}^{n+1-\alpha} u\right)\right\|_{W_{a+}^{1, p}}^{p} \\
& \left.\quad=M_{1,1}\left(\left\|D^{n}\left(I_{a+}^{n-(\alpha-1)} u\right)\right\|_{L_{p}}^{p}+\| D_{a+}^{\alpha} u\right) \|_{L_{p}}^{p}\right),
\end{aligned}
$$

where $M_{1,1}$ is such that

$$
\|v\|_{a, W_{a+}^{1, p}}^{p} \leq M_{1,1}\|v\|_{W_{a+}^{1, p}}^{p}
$$

Consequently,

$$
\begin{aligned}
\|u\|_{a, W_{a+}^{\alpha, p}}^{p}= & \sum_{i=0}^{n-1}\left|D^{i} I_{a+}^{n-(\alpha-1)} u(a)\right|^{p} \\
& +\left|D^{n} I_{a+}^{n-(\alpha-1)} u(a)\right|^{p}+\left\|D_{a+}^{\alpha} u\right\|_{L^{p}}^{p} \\
\leq & M_{\alpha-1, n}\left(\|u\|_{L^{p}}^{p}+\sum_{i=0}^{n-2}\left\|D_{a+}^{\alpha-1-(n-1)+i} u\right\|_{L^{p}}^{p}\right. \\
& \left.+\left\|D_{a+}^{\alpha-1} u\right\|_{L^{p}}^{p}\right) \\
& +M_{1,1}\left(\left\|D_{a+}^{\alpha-1} u\right\|_{L_{p}^{p}}^{p}+\left\|D_{a+}^{\alpha} u\right\|_{L_{p}}^{p}\right) \\
\leq & M_{\alpha, n+1}\left(\|u\|_{L^{p}}^{p}+\sum_{i=0}^{n-1}\left\|D_{a+}^{\alpha-1-(n-1)+i} u\right\|_{L^{p}}^{p}\right. \\
= & M_{\alpha, n+1}\|u\|_{W_{a+}^{\alpha, p}}^{p} \\
+ & M_{\alpha, n+1}\left(\|u\|_{L^{p}}^{p}+\sum_{i=0}^{\alpha}\left\|D_{a+}^{\alpha-n+i} u\right\|_{L_{p}^{p}}^{p}\right)
\end{aligned}
$$

where $M_{\alpha, n+1}=M_{\alpha-1, n}+M_{1,1}$.

\section{Basic Properties of $W_{a+}^{\alpha, p}$}

Now, we are in a position to prove some basic properties of the introduced spaces.

Theorem 25. The space $W_{a+}^{\alpha, p}$ is complete with respect to each of the norms $\|\cdot\|_{W_{a+}^{\alpha, p}}$ and $\|\cdot\|_{a, W_{a+}^{\alpha, p}}$, for any $\alpha>0$ and $1 \leq p<$ $\infty$.

Proof. Let $n \in \mathbb{N}$ be such that $\alpha \in(n-1, n]$. Of course, it is sufficient to show that $W_{a+}^{\alpha, p}$ with the norm $\|\cdot\|_{a, W_{a+}^{\alpha, p}}$ is complete. Let $\left(u_{k}\right) \subset W_{a+}^{\alpha, p}$ be a Cauchy sequence with respect to this norm. So, the sequences $\left(D^{i} I_{a+}^{n-\alpha} u_{k}(a)\right), i=$ $0, \ldots, n-1$, are Cauchy sequences in $\mathbb{R}^{m}$ and $\left(D_{a+}^{\alpha} u_{k}\right)$ is the Cauchy sequence in $L^{p}$. Let $c_{0}, c_{1}, \ldots, c_{n-1} \in \mathbb{R}^{m}$ and $\varphi \in L^{p}$ be limits of the above sequences in $\mathbb{R}^{m}$ and $L^{p}$, respectively. Then the function

$$
\begin{aligned}
u(t)= & \sum_{i=0}^{n-1} \frac{c_{i}}{\Gamma(\alpha-n+1+i)}(t-a)^{\alpha-n+i} \\
& +I_{a+}^{\alpha} \varphi(t), \quad t \in[a, b] \text { a.e., }
\end{aligned}
$$

belongs to $W_{a+}^{\alpha, p}$ and is the limit of $\left(u_{k}\right)$ in $W_{a+}^{\alpha, p}$ with respect to $\|\cdot\|_{a, W_{a+}^{\alpha, p}}$ (to assert that $u \in L^{p}$ it is sufficient to consider the cases $(n-\alpha) p<1$ and $(n-\alpha) p \geq 1$-in the second case $I_{a+}^{n-\alpha} u_{k}(a)=0$ for any $k \in \mathbb{N}$ and, consequently, $\left.c_{0}=0\right)$.

In the proofs of the next two theorems we use the method presented in [1].

Theorem 26. The space $W_{a+}^{\alpha, p}$ is reflexive with respect to each of the norms $\|\cdot\|_{W_{a+}^{\alpha, p}}$ and $\|\cdot\|_{a, W_{a+}^{\alpha, p}}$, for any $\alpha>0$ and $1<p<$ $\infty$.

Proof. Let us consider $W_{a+}^{\alpha, p}$ with the norm $\|\cdot\|_{W_{a+}^{\alpha, p}}$ and define a mapping

$$
\begin{aligned}
\lambda: W_{a+}^{\alpha, p} \ni u & \longmapsto\left(u, D_{a+}^{\alpha-(n-1)} u, \ldots, D_{a+}^{\alpha-1} u, D_{a+}^{\alpha} u\right) \\
& \in L^{p} \times L^{p} \times \cdots \times L^{p} \times L^{p} .
\end{aligned}
$$

Since $\lambda$ is the isometry, $\lambda\left(W_{a+}^{\alpha, p}\right)$ is the closed linear subspace of the reflexive space $L^{p} \times \cdots \times L^{p}$. So (cf. [8, Corollary 1 in Part V.73]), it is reflexive and, consequently, $W_{a+}^{\alpha, p}$ is reflexive with respect to $\|\cdot\|_{W_{a+}^{\alpha, p}}$.

From the equivalence of the norms it follows that $W_{a+}^{\alpha, p}$ with the norm $\|\cdot\|_{a, W_{a+}^{\alpha, p}}$ is also reflexive (it is sufficient to consider the identity mapping $i:\left(W_{a+}^{\alpha, p},\|\cdot\|_{W_{a+}^{\alpha, p}}\right) \rightarrow$ $\left(W_{a+}^{\alpha, p},\|\cdot\|_{W_{a+}^{\alpha, p}}\right)$ being the linear homeomorphism and use [8, Remark in Part V.7.3]).

Theorem 27. The space $W_{a+}^{\alpha, p}$ is separable with respect to each of the norms $\|\cdot\|_{W_{a+}^{\alpha, p}}$ and $\|\cdot\|_{a, W_{a+}^{\alpha, p}}$, for any $\alpha>0$ and $1 \leq p<$ $\infty$.

Proof. Let us consider $W_{a+}^{\alpha, p}$ with the norm $\|\cdot\|_{W_{a+}^{\alpha, p}}$ and mapping $\lambda$ defined in the proof of Theorem 26. Of course, $\lambda\left(W_{a+}^{\alpha, p}\right)$ is separable as a subset of separable space $L^{p} \times \cdots \times L^{p}$. Since $\lambda$ is the isometry, $W_{a+}^{\alpha, p}$ is also separable with respect to the norm $\|\cdot\|_{W_{a+}^{\alpha, p}}$. Equivalence of the norms $\|\cdot\|_{a, W_{a+}^{\alpha, p}}$ and $\|\cdot\|_{W_{a+}^{\alpha, p}}$ implies separability of $W_{a+}^{\alpha, p}$ with respect to $\|\cdot\|_{W_{a+}^{\alpha, p}}$.

\section{Imbeddings}

We have the following extension of Theorem 12 . 
Theorem 28. (a) If $0<\beta<\alpha \leq 1$, then

$$
A C_{a+}^{\alpha, p} \subset I_{a+}^{\beta}\left(L^{q}\right) \subset A C_{a+}^{\beta, q}
$$

for $1 \leq q \leq p<\infty$ and $1 \leq q<1 /(1-\alpha+\beta)$; consequently,

$$
W_{a+}^{\alpha, p} \subset W_{a+}^{\beta, q}
$$

for $1 \leq q \leq p<\infty$ and $1 \leq q<1 /(1-\alpha+\beta)$. then

(b) If $n-1<\alpha \leq n, n \in \mathbb{N}, n \geq 2$, and $0<\beta<\alpha-(n-1)$,

$$
A C_{a+}^{\alpha, p} \subset I_{a+}^{\beta}\left(L^{q}\right) \subset A C_{a+}^{\beta, q}
$$

for $1 \leq p<\infty$ and $1 \leq q<1 /(n-\alpha+\beta)$; consequently,

$$
W_{a+}^{\alpha, p} \subset W_{a+}^{\beta, q}
$$

for $1 \leq p<\infty$ and $1 \leq q<(1 /(n-\alpha+\beta))$.

(c) If $n-1<\alpha \leq n, n \in \mathbb{N}, n \geq 2, \beta=\alpha-i$, and $i \in\{1, \ldots, n-1\}$, then

$$
A C_{a+}^{\alpha, p} \subset A C_{a+}^{\beta, q}
$$

for $1 \leq p<\infty$ and $1 \leq q<\infty$; consequently,

$$
W_{a+}^{\alpha, p} \subset W_{a+}^{\beta, q}
$$

for $1 \leq p<\infty,(n-\alpha) p<1,1 \leq q<\infty$, and $(n-\alpha) q<1$ or $1 \leq p<\infty,(n-\alpha) p \geq 1$, and $1 \leq q<\infty$.

(d) If $0<\alpha=\beta$, then

$$
A C_{a+}^{\alpha, p} \subset A C_{a+}^{\beta, q}
$$

for $1 \leq q \leq p<\infty$; consequently,

$$
W_{a+}^{\alpha, p} \subset W_{a+}^{\alpha, q}
$$

for $1 \leq q \leq p<\infty$.

Proof. (a) Let us fix $p \in[1, \infty)$. Theorems 12 and 13 (a) imply that

$$
A C_{a+}^{\alpha, p} \subset A C_{a+}^{\alpha, 1} \subset I_{a+}^{\beta}\left(L^{q}\right) \subset A C_{a+}^{\beta, q}
$$

provided that $(\alpha-\beta-1) q>-1$ and $1 \leq q \leq p$ (cf. (20), (21)); that is, $1 \leq q<1 /(1-\alpha+\beta)$ and $1 \leq q \leq p$. Consequently, for such $p$ and $q$,

$$
W_{a+}^{\alpha, p}=A C_{a+}^{\alpha, p} \cap L^{p} \subset A C_{a+}^{\beta, q} \cap L^{q}=W_{a+}^{\beta, q} .
$$

(b) Let us fix $p \in[1, \infty)$. In this case $\alpha-\beta>1$ and, consequently, $I_{a+}^{\alpha-\beta} \varphi=I_{a+}^{1} I_{a+}^{\alpha-\beta-1} \varphi \in C$ for any $\varphi \in L^{1}$. So (cf. Theorems 12 and 13 (a))

$$
A C_{a+}^{\alpha, p} \subset A C_{a+}^{\alpha, 1} \subset I_{a+}^{\beta}\left(L^{q}\right) \subset A C_{a+}^{\beta, q}
$$

provided that $(\alpha-\beta-n) q>-1$ and $1 \leq q<\infty$ (cf. (20), (21)); that is, $1 \leq q<1 /(n-\alpha+\beta)$. Consequently, for such $q$ and $1 \leq p<\infty$,

$$
\begin{aligned}
W_{a+}^{\alpha, p} & =A C_{a+}^{\alpha, p} \cap L^{p} \subset I_{a+}^{\beta}\left(L^{q}\right) \\
& =I_{a+}^{\beta}\left(L^{q}\right) \cap L^{q} \subset A C_{a+}^{\beta, q} \cap L^{q}=W_{a+}^{\beta, q}
\end{aligned}
$$

(we used here the inclusion $I_{a+}^{\beta}\left(L^{q}\right) \subset L^{q}$ ).

(c) Let us fix $p \in[1, \infty)$. Theorems 12 and 13 (b) imply that

$$
A C_{a+}^{\alpha, p} \subset A C_{a+}^{\alpha, 1} \subset A C_{a+}^{\beta, q}
$$

for any $q \in[1, \infty)($ cf. $(22),(24))$.

If $(n-\alpha) p<1$, then (cf. Remark 21) $A C_{a+}^{\alpha, p} \cap L^{p}=A C_{a+}^{\alpha, p}$. So,

$$
\begin{aligned}
W_{a+}^{\alpha, p} & =A C_{a+}^{\alpha, p} \cap L^{p}=A C_{a+}^{\alpha, p} \subset A C_{a+}^{\beta, q} \subset A C_{a+}^{\beta, q} \cap L^{q} \\
& =W_{a+}^{\beta, q}
\end{aligned}
$$

provided that (cf. Theorem 13 (b)) $(n-\alpha) q<1$. If $(n-\alpha) p \geq$ 1 , then (cf. Remark 21) $A C_{a+}^{\alpha, p} \cap L^{p}$ is the set of all functions belonging to $A C_{a+}^{\alpha, p}$ that satisfy the condition $\left(I_{a+}^{n-\alpha} f\right)(a)=0$. Consequently (cf. Theorem 13 (b)), $A C_{a+}^{\alpha, p} \cap L^{p} \subset A C_{a+}^{\beta, q} \cap L^{q}$, that is, $W_{a+}^{\alpha, p} \subset W_{a+}^{\beta, q}$ for any $1 \leq q<\infty$.

(d) These facts are obvious.

The first part of the point (c) of the above theorem implies the following.

Corollary 29. If $n-1<\alpha \leq n, n \in \mathbb{N}, n \geq 2, \beta=\alpha-i$, and $i \in\{1, \ldots, n-1\}$, then

$$
W_{a+}^{\alpha, p} \subset W_{a+}^{\beta, q}
$$

for $1 \leq q \leq p<\infty$.

In the next section we will use the following important result obtained in [9, Lemma 1.1].

Theorem 30. If $\alpha>0$ and $1 \leq p<\infty$, then the operator $I_{a+}^{\alpha}: L^{p} \rightarrow L^{p}$ is completely continuous, that is, it maps the bounded sets onto relatively compact ones.

Now, we are in a position to prove theorems on compactness of some imbeddings.

Theorem 31. The imbedding

$$
W_{a+}^{\alpha, p} \subset W_{a+}^{\beta, q}
$$

for $0<\beta<\alpha \leq 1,1 \leq q \leq p<\infty$, and $1 \leq q<1 /(1-\alpha+\beta)$, given in Theorem 28 (a), is compact.

Proof. Let $0<\beta<\alpha \leq 1,1 \leq q \leq p<\infty, 1 \leq q<$ $1 /(1-\alpha+\beta)$, and $\left(u_{k}\right)$, where

$$
\begin{array}{r}
u_{k}(t)=\frac{c_{k}}{\Gamma(\alpha)} \frac{1}{(t-a)^{1-\alpha}}+I_{a+}^{\alpha} \varphi_{k}(t), \\
t \in[a, b] \text { a.e., } k \in \mathbb{N},
\end{array}
$$


be a bounded sequence in $W_{a+}^{\alpha, p}$. We will show that it contains a subsequence which is convergent in $W_{a+}^{\beta, q}$.

Since $\left(u_{k}\right)$ is bounded (cf. Theorem 24 ), the sequences $\left(c_{k}\right)$ and $\left(\varphi_{k}\right)$ are bounded in $\mathbb{R}^{m}$ and $L^{p}$, respectively. So, one can choose a subsequence $\left(k_{j}\right)_{j \in \mathbb{N}}$ of positive integers such that $\left(c_{k_{j}}\right)$ is convergent to some $c_{0}$ in $\mathbb{R}^{m}$ and (cf. Theorem 30) such that $\left(I_{a+}^{\alpha-\beta} \varphi_{k_{j}}\right)$ is convergent to some $\bar{\psi}_{0}$ in $L^{p}$. Of course, $\left(I_{a+}^{\alpha-\beta} \varphi_{k_{j}}\right)$ is convergent to $\bar{\psi}_{0}$ in $L^{q}$. Moreover, the sequence $\left(\left(c_{k_{j}} / \Gamma(\alpha-\beta)\right)(\cdot-a)^{\alpha-1-\beta}\right)$ converges in $L^{q}$ to $\left(c_{0} / \Gamma(\alpha-\beta)\right)(\cdot-$ $a)^{\alpha-1-\beta}$. This means (cf. (20), (21)) that the sequence $\left(u_{k_{j}}\right)$ converges in $W_{a+}^{\beta, q}$ to $u_{0}$ given by

$$
u_{0}(t)=I_{a+}^{\beta} \psi_{0}(t), \quad t \in[a, b] \text { a.e., }
$$

where $\psi_{0}(t)=\left(c_{0} / \Gamma(\alpha-\beta)\right)(t-a)^{\alpha-1-\beta}+\bar{\psi}_{0}(t)$. The proof is completed.

The above theorem implies the following.

\section{Corollary 32. The imbedding}

$$
W_{a+}^{\alpha, p} \subset L^{q}
$$

for $0<\alpha<1,1 \leq q \leq p<\infty, 1 \leq q<1 /(1-\alpha)$ or $\alpha=1$, and $1 \leq q \leq p<\infty$ is compact.

Theorem 33. The imbedding

$$
W_{a+}^{\alpha, p} \subset W_{a+}^{\beta, q}
$$

for $n-1<\alpha \leq n, n \in \mathbb{N}, n \geq 2,0<\beta<\alpha-(n-1), 1 \leq p<\infty$, and $1 \leq q<1 /(n-\alpha+\beta)$, given in Theorem $28(b)$, is compact.

Proof. Let us consider a bounded sequence $\left(u_{k}\right)$ in $W_{a+}^{\alpha, p}$ where

$$
\begin{aligned}
u_{k}(t)= & \frac{c_{0}^{k}}{\Gamma(\alpha-n+1)}(t-a)^{\alpha-n} \\
& +\frac{c_{1}^{k}}{\Gamma(\alpha-n+2)}(t-a)^{\alpha-n+1} \\
& +\cdots+\frac{c_{n-1}^{k}}{\Gamma(\alpha)}(t-a)^{\alpha-1} \\
& +I_{a+}^{\alpha} \varphi_{k}(t), \quad t \in[a, b] \text { a.e., }
\end{aligned}
$$

with $c_{0}^{k}, c_{1}^{k}, \ldots, c_{n-1}^{k} \in \mathbb{R}^{m}$ and $\varphi_{k} \in L^{p}$. We will show that it contains a subsequence which is convergent in $W_{a+}^{\beta, q}$.

Since $\left(u_{k}\right)$ is bounded, the sequences $\left(c_{0}^{k}\right),\left(c_{1}^{k}\right), \ldots,\left(c_{n-1}^{k}\right) \in \mathbb{R}^{m}$ and $\left(\varphi_{k}\right)$ are bounded in $\mathbb{R}^{m}$ and $L^{p}$, respectively. So, one can choose a subsequence $\left(k_{j}\right)_{j \in \mathbb{N}}$ of positive integers such that $\left(c_{0}^{k_{j}}\right),\left(c_{1}^{k_{j}}\right), \ldots,\left(c_{n-1}^{k_{j}}\right)$ are convergent to some $c_{0}, c_{1}, \ldots, c_{n-1}$ in $\mathbb{R}^{m}$ and (cf. Theorem 30) such that the sequence $\left(I_{a+}^{\gamma} \varphi_{k_{j}}\right)$ is convergent in $L^{p}$ to some $\omega_{0}$ where $\gamma=(\alpha-\beta)-1>0$. Consequently, $\left(I_{a+}^{\gamma} \varphi_{k_{j}}\right)$ is convergent in $L^{1}$ to $\omega_{0}$ and

$$
I_{a+}^{\alpha-\beta} \varphi_{k_{j}}=I_{a+}^{1} I_{a+}^{\gamma} \varphi_{k_{j}} \longrightarrow I_{a+}^{1} \omega_{0}
$$

in $L^{q}$, because

$$
\begin{aligned}
& \int_{a}^{b}\left|I_{a+}^{1} I_{a+}^{\gamma} \varphi_{k_{j}}(t)-I_{a+}^{1} \omega_{0}(t)\right|^{q} d t \\
& \quad \leq \int_{a}^{b}\left(\int_{a}^{t}\left|I_{a+}^{\gamma} \varphi_{k_{j}}(s)-\omega_{0}(s)\right| d s\right)^{q} d t \\
& \quad \leq\left\|I_{a+}^{\gamma} \varphi_{k_{j}}-\omega_{0}\right\|_{L^{1}}^{q}(b-a) .
\end{aligned}
$$

So, the sequence $\left(D_{a+}^{\beta} u_{k_{j}}\right)$, where

$$
\begin{aligned}
D_{a+}^{\beta} u_{k_{j}}(t)= & \frac{c_{0}^{k_{j}}}{\Gamma(\alpha-n+1-\beta)}(t-a)^{\alpha-n-\beta} \\
& +\frac{c_{1}^{k_{j}}}{\Gamma(\alpha-n+2-\beta)}(t-a)^{\alpha-n-\beta+1} \\
& +\cdots+\frac{c_{n-1}^{k_{j}}}{\Gamma(\alpha-\beta)}(t-a)^{\alpha-\beta-1}+I_{a+}^{\alpha-\beta} \varphi_{k_{j}}(t),
\end{aligned}
$$

converges to the function

$$
\begin{aligned}
\psi_{0}(t)= & \frac{c_{0}}{\Gamma(\alpha-n+1-\beta)}(t-a)^{\alpha-n-\beta} \\
& +\frac{c_{1}}{\Gamma(\alpha-n+2-\beta)}(t-a)^{\alpha-n-\beta+1} \\
& +\cdots+\frac{c_{n-1}}{\Gamma(\alpha-\beta)}(t-a)^{\alpha-\beta-1} \\
& +I_{a+}^{1} \omega_{0}(t)
\end{aligned}
$$

in $L^{q}$. Thus, the sequence $\left(u_{k_{j}}\right)$ converges in $W_{a+}^{\beta, q}$ to $I_{a+}^{\beta} \psi_{0}$. The proof is completed.

The above theorem implies the following.

Corollary 34. The imbedding

$$
W_{a+}^{\alpha, p} \subset L^{q}
$$

for $n-1<\alpha<n, n \in \mathbb{N}, n \geq 2,1 \leq p<\infty, 1 \leq q<1 /(n-\alpha)$ or $\alpha=n, n \in \mathbb{N}, n \geq 2,1 \leq p<\infty$, and $1 \leq q<\infty$ is compact.

Theorem 35. The imbedding

$$
W_{a+}^{\alpha, p} \subset W_{a+}^{\beta, q},
$$

for $n-1<\alpha \leq n, n \in \mathbb{N}, n \geq 2, \beta=\alpha-i, i \in\{1, \ldots, n-1\}$, and $1 \leq q \leq p<\infty$, given in Corollary 29, is compact. 
Proof. Let us consider a bounded sequence $\left(u_{k}\right)$ in $W_{a+}^{\alpha, p}$ where

$$
\begin{aligned}
u_{k}(t)= & \frac{c_{0}^{k}}{\Gamma(\alpha-n+1)}(t-a)^{\alpha-n} \\
& +\frac{c_{1}^{k}}{\Gamma(\alpha-n+2)}(t-a)^{\alpha-n+1} \\
& +\cdots+\frac{c_{n-1}^{k}}{\Gamma(\alpha)}(t-a)^{\alpha-1} \\
& +I_{a+}^{\alpha} \varphi_{k}(t), \quad t \in[a, b] \text { a.e., }
\end{aligned}
$$

with $c_{0}^{k}, c_{1}^{k}, \ldots, c_{n-1}^{k} \in \mathbb{R}^{m}$ and $\varphi_{k} \in L^{p}$. We will show that it contains a subsequence which is convergent in $W_{a+}^{\beta, q}$.

Since $\left(u_{k}\right)$ is bounded, the sequences $\left(c_{0}^{k}\right),\left(c_{1}^{k}\right), \ldots$, $\left(c_{n-1}^{k}\right) \in \mathbb{R}^{m}$ and $\left(\varphi_{k}\right)$ are bounded in $\mathbb{R}^{m}$ and $L^{p}$, respectively. So, one can choose a subsequence $\left(k_{j}\right)_{j \in \mathbb{N}}$ of positive integers such that $\left(c_{0}^{k_{j}}\right),\left(c_{1}^{k_{j}}\right), \ldots,\left(c_{n-1}^{k_{j}}\right)$ are convergent to some $c_{0}, c_{1}, \ldots, c_{n-1}$ in $\mathbb{R}^{m}$ and (cf. Theorem 30 ) such that

$$
\left(I_{a+}^{1} c_{n-i+1}^{k_{j}}\right), \ldots,\left(I_{a+}^{i-1} c_{n-1}^{k_{j}}\right),\left(I_{a+}^{i} \varphi_{k_{j}}\right)
$$

are convergent to some $\bar{\psi}_{1,0}, \ldots, \bar{\psi}_{i-1,0}, \bar{\psi}_{0}$ in $L^{p}$ and, consequently, in $L^{q}$. So (cf. Theorem 13 (b)),

$$
\begin{aligned}
D_{a+}^{\beta} u_{k_{j}} & =c_{n-i}^{k_{j}}+I_{a+}^{1} c_{n-i+1}^{k_{j}}+\cdots+I_{a+}^{i-1} c_{n-1}^{k_{j}}+I_{a+}^{i} \varphi_{k_{j}} \\
& \longrightarrow \psi_{0}
\end{aligned}
$$

in $L^{q}$, where $\psi_{0} \in L^{q}$ is given by

$$
\begin{array}{r}
\psi_{0}(t)=c_{n-i}+\bar{\psi}_{1,0}(t)+\cdots+\bar{\psi}_{i-1,0}(t)+\bar{\psi}_{0}(t), \\
t \in[a, b] \text { a.e. }
\end{array}
$$

This means (cf. (22), (23), and (24)) that the sequence $\left(u_{k_{j}}\right)$ converges in $W_{a+}^{\beta, q}$ to $u_{0}$ given by

$$
\begin{aligned}
u_{0}(t)= & \frac{d_{0}}{\Gamma(\beta-(n-i)+1)}(t-a)^{\beta-(n-i)} \\
& +\frac{d_{1}}{\Gamma(\beta-(n-i)+2)}(t-a)^{\beta-(n-i)+1} \\
& +\cdots+\frac{d_{n-(i+1)}}{\Gamma(\beta)}(t-a)^{\beta-1} \\
& +I_{a+}^{\beta} \psi_{0}(t), \quad t \in[a, b] \text { a.e., }
\end{aligned}
$$

where

$$
d_{0}=c_{0}, \quad d_{1}=c_{1}, \ldots, \quad d_{n-(i+1)}=c_{n-(i+1)} .
$$

The proof is completed.

The above theorem implies the following.
Corollary 36. The imbedding

$$
W_{a+}^{\alpha, p} \subset L^{q}
$$

for $n-1<\alpha \leq n, n \in \mathbb{N}, n \geq 2$, and $1 \leq q \leq p<\infty$ is compact.

Remark 37. It is easy to see that imbeddings $W_{a+}^{\alpha, p} \subset W_{a+}^{\alpha, q}$ for $\alpha>0$ and $1 \leq q \leq p<\infty$, given in Theorem 28 (d), are not compact.

Remark 38. From Corollary 32 it follows that the imbedding $W^{1, p} \subset L^{p}$ is compact for any $1 \leq p<\infty$. Corollary 34 implies the compactness of the imbedding $W^{n, 1} \subset L^{q}$ for any $n \in \mathbb{N}, n \geq 2$, and $1 \leq q<\infty$. The following problem is open: is it possible to strengthen Theorem 31 or Theorem 33 to deduce the compactness of the imbedding $W^{1,1} \subset L^{q}$ for any $1 \leq q<\infty$ ?

\section{Application to Boundary Value Problems}

In this section, we will demonstrate an application of the obtained results to fractional boundary value problems.

Namely, let us fix $\alpha \in(1 / 2,1)$ and consider the following problem:

$$
\begin{gathered}
D_{b-}^{\alpha} D_{a+}^{\alpha} x(t)+x(t)=f(t), \quad t \in[a, b] \text { a.e. } \\
D_{a+}^{\alpha} x(a)=0, \quad x(b)=0,
\end{gathered}
$$

where $f \in L^{2}$. By a solution to this problem we mean a function $x \in W_{a+}^{\alpha, 2}$ such that $D_{a+}^{\alpha} x$ and $D_{b-}^{\alpha} D_{a+}^{\alpha} x$ exist, and satisfying the above equation and boundary conditions. Under the assumption on $\alpha$ the boundary conditions make sense $([10$, Property 4$])$.

Let $a: H \times H \rightarrow \mathbb{R}$, where

$$
H=\left\{x \in W_{a+}^{\alpha, 2} ; x(b)=0\right\}
$$

to be in the following bilinear form

$$
a(x, y)=\int_{a}^{b} D_{a+}^{\alpha} x(t) D_{a+}^{\alpha} y(t) d t+\int_{a}^{b} x(t) y(t) d t
$$

It is easy to see that $H$ is the closed subspace of the Hilbert space $\left(W_{a+}^{\alpha, 2},\|\cdot\|_{W_{a+}^{\alpha, p}}\right)$; it is sufficient to observe that it is closed in $W_{a+}^{\alpha, 2}$ with respect to $\|\cdot\|_{a, W_{a+}^{\alpha, p}}$ and to use Theorem 24 . Of course, $a$ is a scalar product in $H$ and the norm generated by $a$ is simply the norm $\|\cdot\|_{W_{a+}^{\alpha, 2}}$ restricted to $H$. Clearly, $a$ is continuous and coercive; that is, there exists a constant $c>0$ such that $a(x, x) \geq c\|x\|_{W_{a+}^{\alpha, 2}}^{2}$ for $x \in W_{a+}^{\alpha, 2}$; in fact $a(x, x)=$ $\|x\|_{W_{a+}^{\alpha, 2}}^{2}$ for $x \in H$. So, Lax-Milgram theorem [1] or simply 
Riesz-Frechet theorem implies that there exists $x \in W_{a+}^{\alpha, 2}$ such that

$$
\begin{array}{r}
a(x, h)=\int_{a}^{b} f(t) h(t) d t, \quad h \in H, \\
\frac{1}{2}\|x\|_{W_{a+}^{\alpha, 2}}^{2}-\int_{a}^{b} f(t) x(t) d t \\
=\min _{h \in H}\left\{\frac{1}{2}\|h\|_{W_{a+}^{\alpha, 2}}^{2}-\int_{a}^{b} f(t) h(t) d t\right\} .
\end{array}
$$

Condition (135) means that

$$
\int_{a}^{b} D_{a+}^{\alpha} x(t) D_{a+}^{\alpha} h(t) d t=\int_{a}^{b}(f(t)-x(t)) h(t) d t, \quad h \in H .
$$

From a counterpart of Theorem 20 for the weak right fractional derivative it follows that $D_{a+}^{\alpha} x \in W_{b-}^{\alpha, 2}$ and

$$
D_{b-}^{\alpha} D_{a+}^{\alpha} x(t)+x(t)=f(t), \quad t \in[a, b] \text { a.e. }
$$

Since $x \in H, x(b)=0$. Moreover, applying Theorem 17 to the left side of (137) we obtain

$$
\begin{aligned}
& \int_{a}^{b}\left(D_{b-}^{\alpha} D_{a+}^{\alpha} x(t)+x(t)-f(t)\right) h(t) d t \\
& \quad-I_{a+}^{1-\alpha} h(a) D_{a+}^{\alpha} x(a)+I_{b-}^{1-\alpha} D_{a+}^{\alpha} x(b) h(b) \\
& \quad=0
\end{aligned}
$$

for $h \in H$. So,

$$
I_{a+}^{1-\alpha} h(a) D_{a+}^{\alpha} x(a)=0, \quad h \in H .
$$

This means that $D_{a+}^{\alpha} x(a)=0$. Indeed, it is sufficient to consider functions $h_{i}, i=1, \ldots, m$, of the form

$$
\begin{aligned}
h_{i}(t)=\left(0, \ldots, 0, \frac{1}{\Gamma(\alpha)} \frac{c}{(t-a)^{1-\alpha}}\right. \\
\left.\quad+\frac{1}{\Gamma(\alpha)} \int_{a}^{t} \frac{1}{(t-\tau)^{1-\alpha}}, 0, \ldots, 0\right),
\end{aligned}
$$

where $c=-(b-a)^{1-\alpha} \int_{a}^{b}\left(1 /(b-\tau)^{1-\alpha}\right) d t$, with nonzero $i$ th coordinate function (of course, $h_{i} \in H$ and $I_{a+}^{1-\alpha} h_{i}(a)=$ $(0, \ldots, 0, c, 0 \ldots, 0) \neq 0)$.

Remark 39. In the same way one can prove the existence of a solution to system

$$
D_{b-}^{\alpha} D_{a+}^{\alpha} x(t)+x(t)=f(t), \quad t \in[a, b] \text { a.e. }
$$

with boundary conditions

$$
I_{a+}^{1-\alpha} x(a)=0, \quad I_{b-}^{1-\alpha} D_{a+}^{\alpha} x(b)=0
$$

or

$$
I_{a+}^{1-\alpha} x(a)=0, \quad x(b)=0,
$$

in the space $W_{a+}^{\alpha, 2}$. In [4], system (142) with nonhomogeneous boundary conditions

$$
I_{a+}^{1-\alpha} x(a)=C, \quad x(b)=D,
$$

where $C, D \in \mathbb{R}^{m}$, is investigated using the Stampacchia theorem (cf. [1]). Nonlinear system of the form

$$
D_{b-}^{\alpha} D_{a+}^{\alpha} x(t)=-F_{x}(t, x(t)), \quad t \in[a, b] \text { a.e., }
$$

where $F_{x}$ is the gradient (in $x$ ) of a potential $F=F(t, x)$, with the above boundary conditions can be studied using the direct method of calculus of variations (cf. [11] for the case of (144)).

It is worth noting that if $\alpha \in(1 / 2,1), x \in W_{a+}^{\alpha, 2}$, and $I_{a+}^{1-\alpha} x(a)=0$, then $x(a)=0$. It follows from the integral representation of $x$ and from the fact that $I_{a+}^{\alpha} f$ is Hölder continuous on $(a, b]$ and $\lim _{t \rightarrow a^{+}} I_{a+}^{\alpha} f(t)=0$ when $f \in L^{2}$ and $\alpha \in(1 / 2,1)$ (cf. [10, Property 4]). So, it is natural to put in such a case $x(a)=0$. It seems that the most accurate functions for investigating the above systems with boundary conditions involving condition $x(a)=0$ (or more general $x(a)=C)$, in general case of $\alpha \in(0,1)$, are the functions possessing the fractional derivatives in Caputo sense; on such functions one assumes that they are absolutely continuous on $[a, b]$ and, consequently, condition $x(a)=0$ makes sense. To our best knowledge, fractional Sobolev spaces via Caputo derivatives have not been investigated up to now and are an open problem.

Remark 40. From the condition (136) it follows that one can search approximate solutions to (132) using numerical methods, for example, the gradient or projection of gradient methods applied to the functional

$$
h \longmapsto \frac{1}{2}\|h\|_{W_{a+}^{\alpha, 2}}^{2}-\int_{a}^{b} f(t) h(t) d t
$$

defined on $H$ and $W_{a+}^{\alpha, 2}$, respectively.

\section{Conflict of Interests}

The authors declare that there is no conflict of interests regarding the publication of this paper.

\section{Acknowledgment}

The project was financed with funds of National Science Centre, granted on the basis of decision DEC-2011/ 01/B/ST7/03426.

\section{References}

[1] H. Brezis, Analyse Fonctionnelle, Theorie et Applications, Masson, Paris, France, 1983.

[2] R. A. Adams, Sobolev Spaces, Academic Press, New York, Ny, USA, 1975.

[3] S. G. Samko, A. A. Kilbas, and O. I. Marichev, Fractional Integrals and Derivatives-Theory and Applications, Gordon and Breach Science Publishers, Amsterdam, The Netherlands, 1993. 
[4] L. Bourdin and D. Idczak, "Fractional fundamental lemma and fractional integration by parts formula-applications to critical points of Bolza functionals and to linear boundary value problems," submitted.

[5] D. Idczak and M. Majewski, "Fractional fundamental lemma of order $\alpha \in(n-1 / 2, n)$ with $n \in N, n \geq 2$," Dynamic Systems and Applications, vol. 21, no. 2-3, pp. 251-268, 2012.

[6] D. Idczak and S. Walczak, "A fractional imbedding theorem," Fractional Calculus and Applied Analysis, vol. 15, no. 3, pp. 418425, 2012.

[7] D. Idczak and S. Walczak, "Compactness of fractional imbeddings," in Proceedings of the 17th International Conference on Methods \& Models in Automation \& Robotics (MMAR '12), pp. 585-588, 2012.

[8] L. W. Kantorowitch and G. P. Akilov, Functional Analysis, Science, Moscow, Russia, 1984, (Russian).

[9] M. W. Michalski, "Derivatives of noninteger order and their applications," in Dissertationes Mathematicae, vol. 338, Polish Academy of Sciences, Warsaw, Poland, 1993.

[10] L. Bourdin, "Existence of a weak solution for fractional EulerLagrange equations," Journal of Mathematical Analysis and Applications, vol. 399, no. 1, pp. 239-251, 2013.

[11] R. Kamocki and M. Majewski, "On a fractional Dirichlet problem," in Proceedings of the 17th International Conference on Methods \& Models in Automation \& Robotics (MMAR '12), pp. 60-63, 2012. 


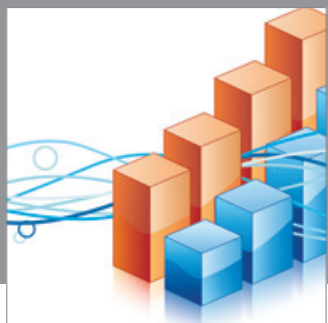

Advances in

Operations Research

mansans

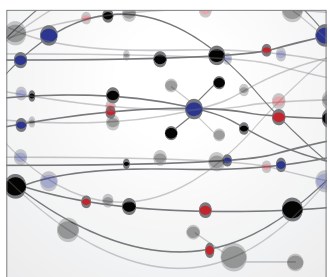

The Scientific World Journal
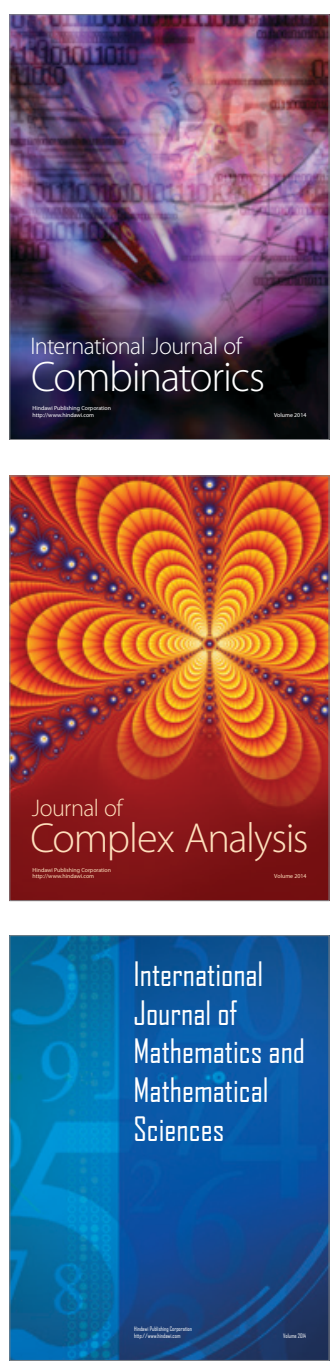
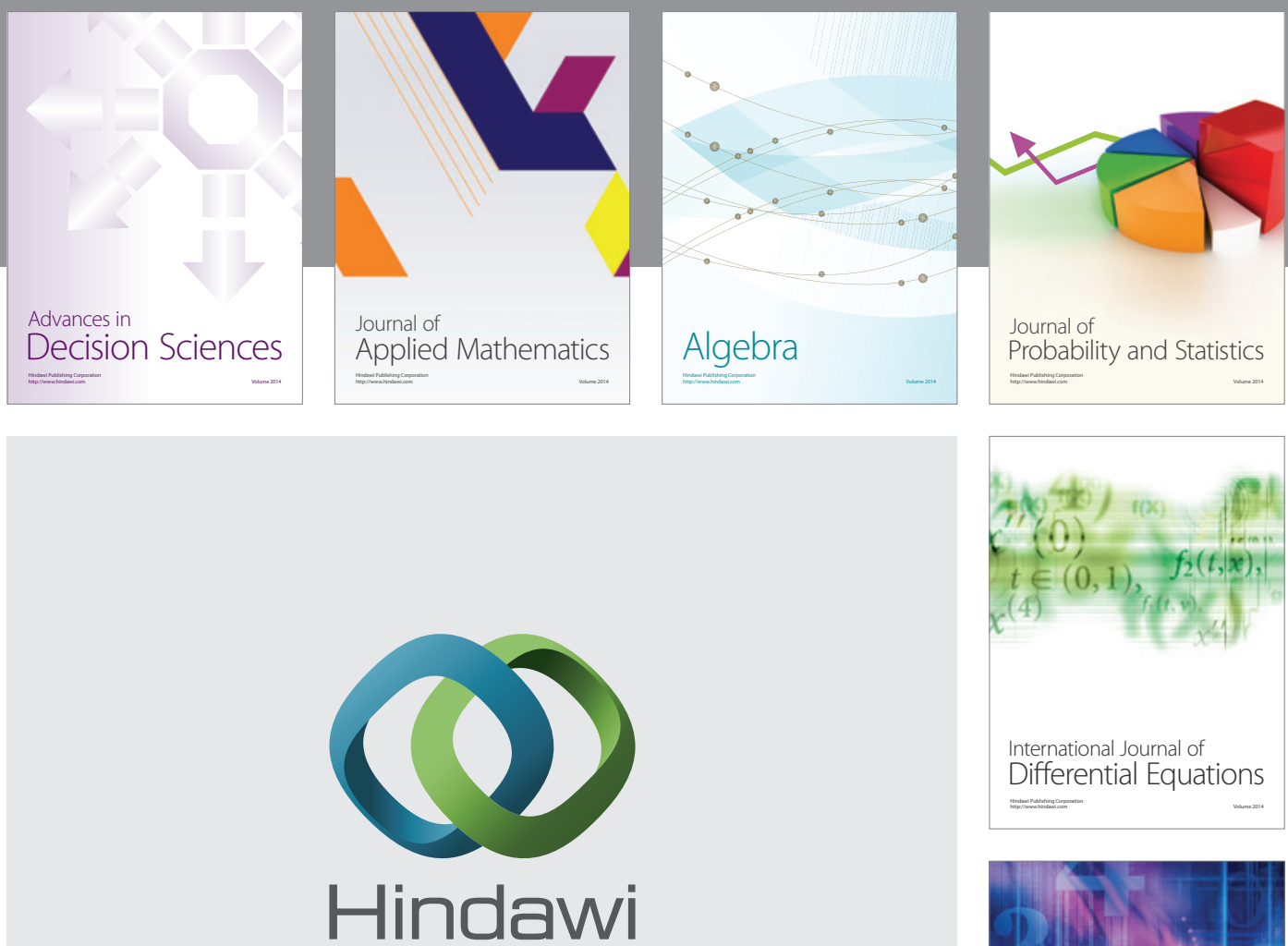

Submit your manuscripts at http://www.hindawi.com
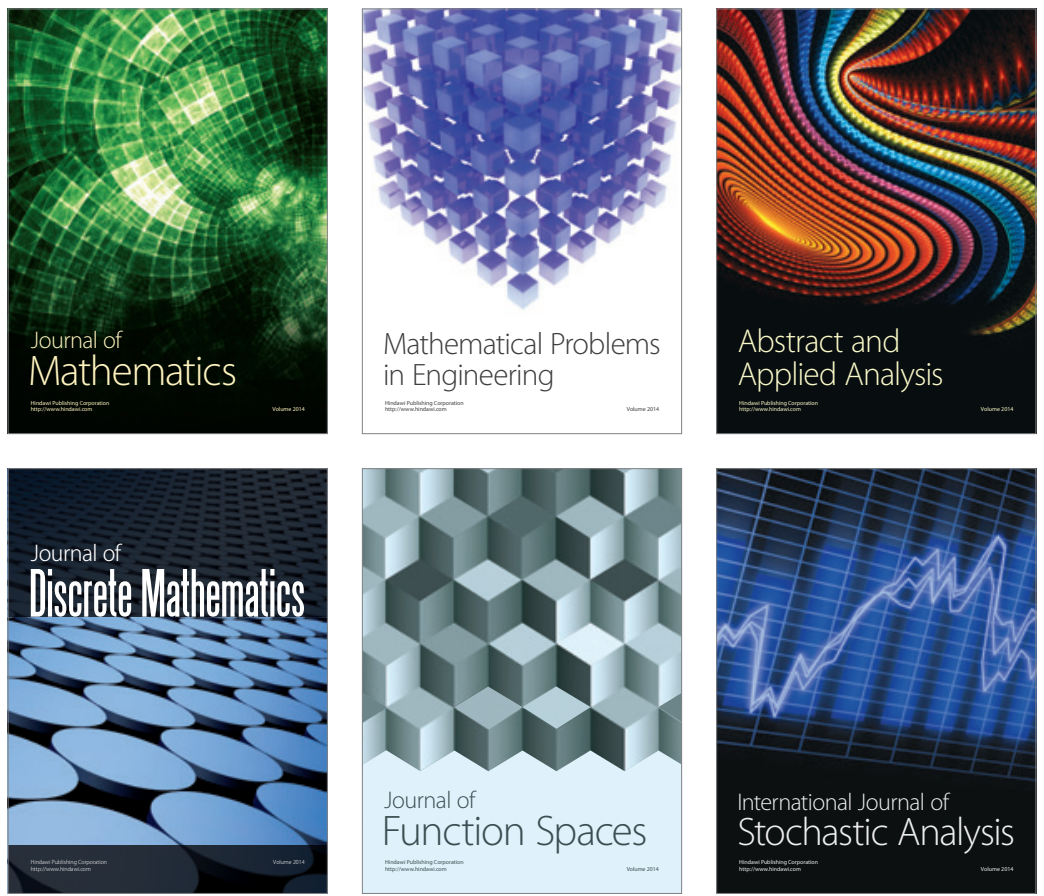

Journal of

Function Spaces

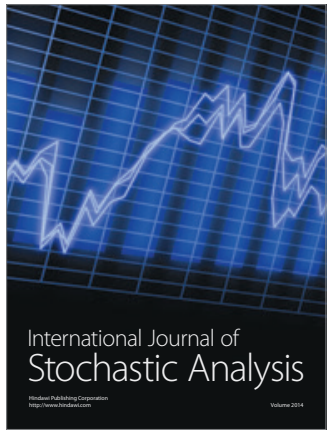

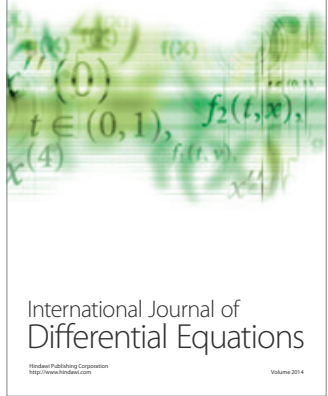
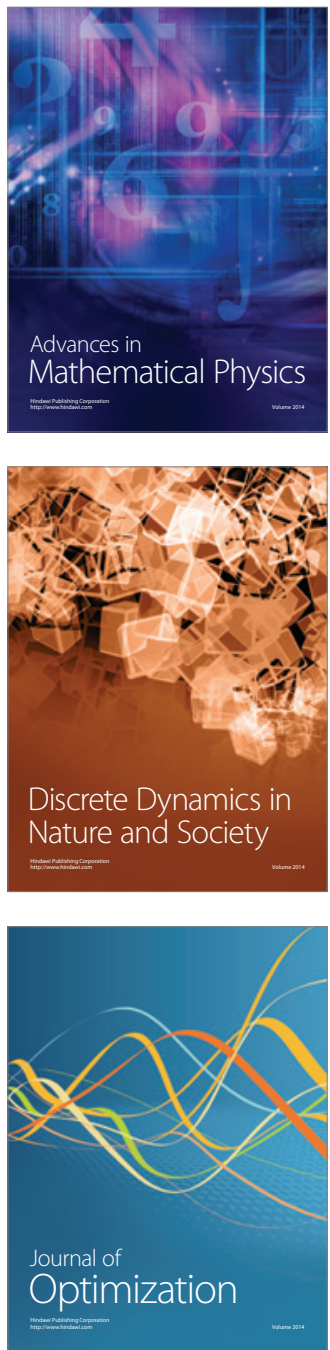